\title{
The Ibaté paleolake in SE Brazil: Record of an exceptional late Santonian palynoflora with multiple significance (chronostratigraphy, paleoecology and paleophytogeography)
}

\author{
Mitsuru Arai ${ }^{a}$, Dimas Dias-Brito ${ }^{\mathrm{b}, *}$ \\ ${ }^{a}$ UNESP - Universidade Estadual Paulista/ IGCE/ UNESPetro - Centro de Geociências aplicadas ao Petróleo, CP 178, CEP 13506-900, Rio Claro, SP, Brazil \\ (Visiting Researcher - PRH 05/ UNESP-ANP) \\ ${ }^{\mathrm{b}}$ UNESP - Universidade Estadual Paulista/ IGCE-DGA/ UNESPetro - Centro de Geociências aplicadas ao Petróleo, CP 178, CEP 13506-900, Rio Claro, SP, \\ Brazil
}

\section{A R T I C L E I N F O}

\section{Article history:}

Received 16 March 2017

Received in revised form

9 November 2017

Accepted in revised form 20 November 2017

Available online 27 November 2017

\section{Keywords:}

Palynology

Anacolosidites eosenonicus palynozone

Paleoclimatology

São Carlos Formation

Paraná Basin

Bauru Group

\begin{abstract}
A B S T R A C T
The Cretaceous Bauru Group of the Paraná Basin in Brazil is a widespread continental unit well known for its vertebrate and invertebrate fossiliferous content. The São Carlos Formation is an exception among its distinct and dominantly reddish siliciclastic units, which are otherwise devoid of palynomorphs. It includes an unique unit, the Ibaté Bed, which corresponds to the lower interval of the Fazenda Nossa Senhora de Fátima Member (FNSF Mbr) and is represented by a dark grey organic silty-argillaceous rhythmite with a rich palynoflora. These rocks are the main focus of this study. Samples were collected from the type section of the formation that is located in the central area of the São Paulo State. Identified palynomorph types are as follows: 32 pteridophyte spore taxa, 31 gymnosperm pollen taxa and 64 angiosperm pollen taxa. Acritarchs, prasinophytes and phytoclasts were also recognized. Seven new taxa are proposed: Anacolosidites eosenonicus sp. nov., Leiosphaeridia ibateensis sp. nov., Stellatia gen. nov., Trichomites brevifurcatus gen. et sp. nov., Trichomites duplihelicoidus gen. et sp. nov., Trichomites simplex gen. et sp. nov. and Tricolpites joelcastroi sp. nov. In quantitative terms, the ephedroid pollen grains - e.g., Equisetosporites, Gnetaceaepollenites and Steevesipollenites - are predominant. Their occurrence associated with Classopollis grains suggest a warm climate tending towards dryness. Some levels are relatively rich in pteridophyte spores, suggestive of contribution from lakeside vegetation. The permanent presence of freshwater is attested by conspicuous microalgal remains (Botryococcus, Pediastrum, Staurastrum and prasinophycean phycomata), recognizable via fluorescence microscopy. Significant amounts of microalgal remains in some samples suggest episodic blooms in the lake, probably induced by eutrophication. The Ibaté Bed corresponds to a depositional scenario involving a distal lacustrine environment with low-oxygen conditions in its bottom waters. As observed in offshore Brazilian basins, the presence of Anacolosidites eosenonicus sp. nov., combined with the absence of Steevesipollenites nativensis, indicates a late Santonian age for the bed (ca. $84 \mathrm{Ma}$ ). Other associated index-palynomorphs are compatible with this time assignment, which is reinforced by the presence of carbonized sclereids that are associated with the "Great Santonian Wildfire" recorded in coeval marine offshore strata of the Campos and Santos basins. The FNSF Mbr is stratigraphically related to the uppermost part of the Adamantina and Uberaba formations of the Bauru Group. Among all known Brazilian Late Cretaceous palynological continental records, this is the richest one ever detected. Although Late Cretaceous in age, the composition of the Ibaté Bed palynoflora reflects better the mid Cretaceous ASA Paleophytogeographic Province.
\end{abstract}

() 2017 Elsevier Ltd. All rights reserved.

\section{Introduction}

The São Carlos Formation is a thin Upper Cretaceous unit included in the widespread Cretaceous Bauru Group of the Paraná Basin, Brazil (Castro et al., 2002). This continental group is mostly

\footnotetext{
* Corresponding author.

E-mail addresses: mitsuru.arai@gmail.com (M. Arai), dimasdb@rc.unesp.br (D. Dias-Brito).
}

formed by reddish sedimentary rocks, including sandstones, siltstones and shales. In some units (e.g. Adamantina Formation) it contains an important fossil content comprising vertebrates, invertebrates, ostracods and charophyta girogonites (Dias-Brito et al., 2001). The absence of organic-walled microfossils in these rocks is noteworthy since they are highly susceptible to oxidation. The São Carlos Formation, which contains several levels of dark and fine sediments, occurs in a small part of the central area of the São Paulo 
State, Southeastern Brazil. It crops out in only a few known sites in the vicinity of São Carlos and Ibaté towns, where the type section is localized. It is exposed along a creek situated in the Nossa Senhora de Fátima Farm on the border of the São Carlos and Ibaté municipalities (Fig. 1).

According to Castro et al. (2002), the siliciclastic succession of the São Carlos Formation - around $40 \mathrm{~m}$ thick - includes, from the base to the top, three distinct facies: a. a marginal lacustrine sandy interval, the "lower sandstone", lying directly on basalts of Serra Geral Formation (Early Cretaceous, $\sim 130 \mathrm{Ma}$ ); b. a lacustrine argillaceous unit, corresponding to the Fazenda Nossa Senhora de Fátima Member (FNSF Mbr); and c. a fluvial sandy interval, the "upper sandstone”, abruptly overlying the lacustrine sediments (Fig. 2).

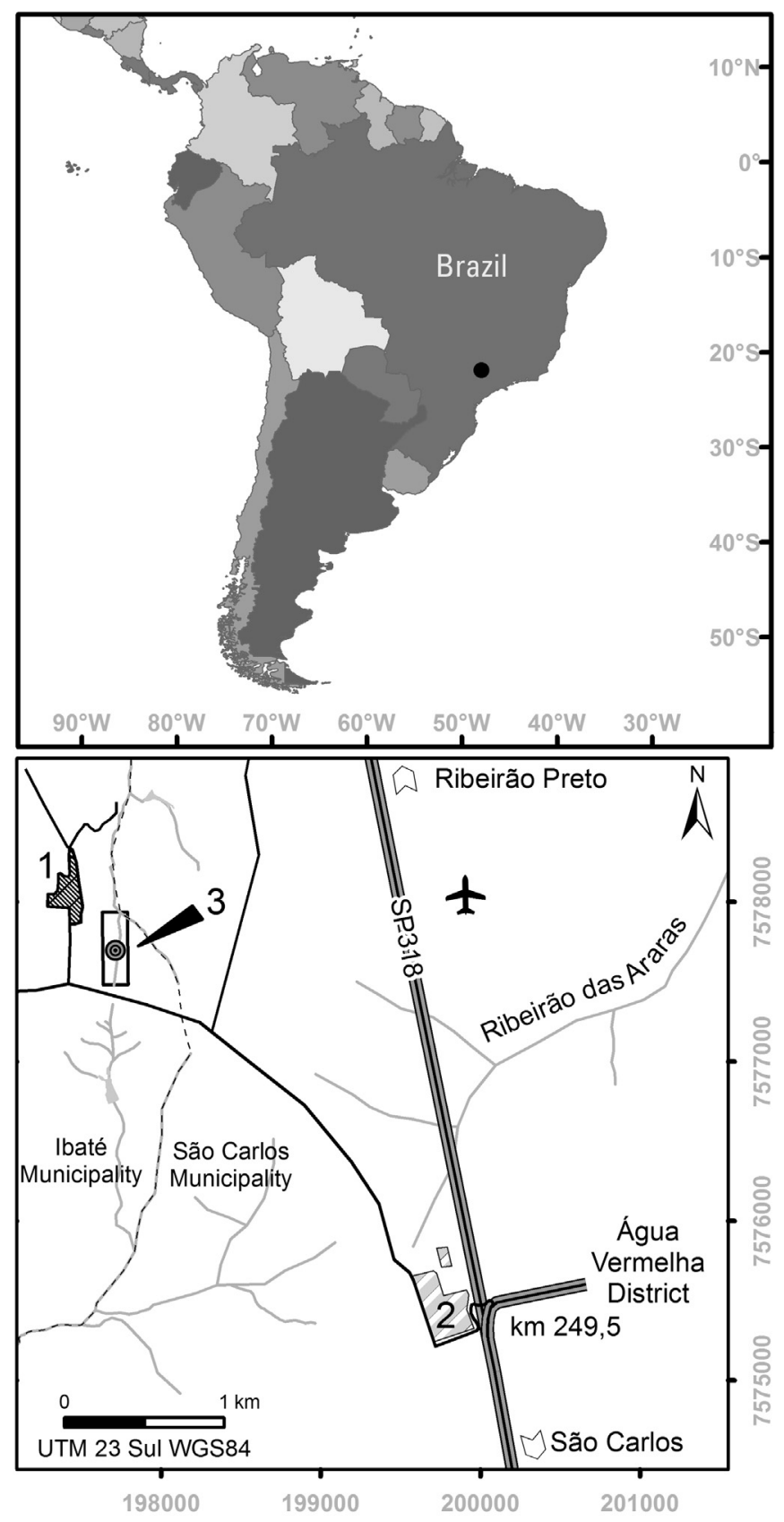

Fig. 1. Outcrop location of the São Carlos Formation type-section. A: geographic position in South America. B: detail of the studied area. 1. Fazenda Nossa Senhora de Fátima Farm headquarters; 2. Factory; 3. Location of the São Carlos Formation typesection.
The lacustrine argillaceous rocks are the focus of this study due to the presence of a rich content of palynomorphs in some levels of the FNSF Mbr, as was previously indicated in Castro et al. (2002). Here we provide an exhaustive documentation of its palynological content, including a list of the recognized palynomorphs, description of new taxa and photomicrographs of the more representative elements of the assemblage. In addition, we discuss its significance in terms of stratigraphic age, paleoenvironment, climate and paleophytogeography.

\section{Geological setting}

\subsection{The FNSF $M b r$}

Rocks of the FNSF Mbr were initially studied by Mezzalira (1974) and considered as Cretaceous or Cenozoic deposits; they were referred to as including dark brown to dark grey shaly argillaceous siltstones and reddish argillaceous siltstones. The dark shaly siltstones were first investigated from a palynological point of view by Lima et al. (1986) and dated as Coniacian. However, no photomicrographic documentation was provided. These latter authors assigned these rocks to the Cretaceous Bauru Group.

As originally described by Castro et al. (2002), the FNSF Mbr (8.2 m thick), is composed of three argillaceous intervals (Fig. 2). The lower one, FNSF 1 ( $0.9 \mathrm{~m}$ thick), as well as the upper one, FNSF 3 (3.8 m thick), mostly consist of reddish sandy-argillaceous rhythmites, with some rare dark argillaceous laminae. They correspond to more proximal and shallow lake conditions. Between them, FNSF 2 (3.5 m thick) is composed of silty and argillaceous laminae couplets, and roughly corresponds to the interval studied by Lima et al. (1986); it represents deposition in a more central lacustrine area.

\subsection{The Ibaté Bed}

FNSF 2 contains a significant fossil content, including palynomorphs, ostracods, conchostracans, vertebrate fragments, bivalves, microgastropods, macroscopic plant debris, and fish scales (Mezzalira, 1974, 1989; Lima et al., 1986; Dias-Brito et al., 2001, 2002; Castro et al., 2002; Rohn et al., 2005). Its lower part (1.3 m thick), here named Ibaté Bed, is a dark grey silty-argillaceous rhythmite, richer in organic matter than the $2.2 \mathrm{~m}$ thick upper part of this interval, with TOC frequently above $1.0 \%$ up to $2.5 \%$ (Castro et al., 2002). The Ibate Bed is the most fossiliferous interval of the FNSF Mbr. It yielded a diverse and rich palynological content, which as discussed below is exceptional in the context of the Bauru Group.

\section{Material and methods}

The studied material has been sampled from the type section of the São Carlos Formation (UTM 237577.6 km, 197.75 km). Among 30 collected samples, 17 of them contain a rich palynological assemblage that constitutes the object of this study (Supplementary Table A). All samples were prepared by standard processing methods of Petrobras' Laboratory (Uesugui, 1979) using hydrochloric and hydrofluoric acids to remove respectively carbonate and silicate mineral components. For each palynological slide, palynomorph content was identified taxonomically and counted for the whole slide using both transmitted light and fluorescence microscopy. Only one slide (SC-14) presented more than 300 specimens. Six slides (UNESP-6, SC-7B, SC-8, SC-10, UNESP-4.1, SC-14) containing more than 100 specimens were chosen to make some quantitative analyses. The optical photomicrographs were taken using a Leica DFC310FX camera coupled with a Leica DM 2500P microscope in laboratory of the UNESPetro (Center for Petroleum Geosciences). Around 30 different taxa derived from two samples presenting 

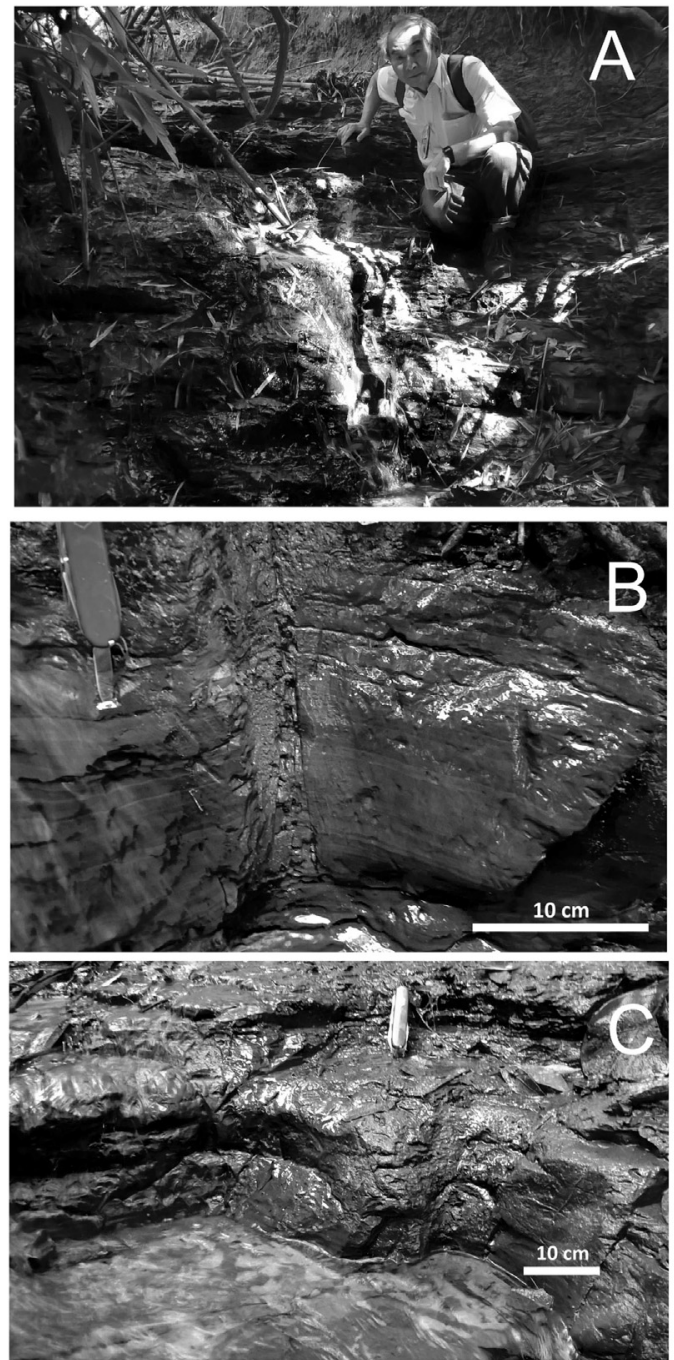

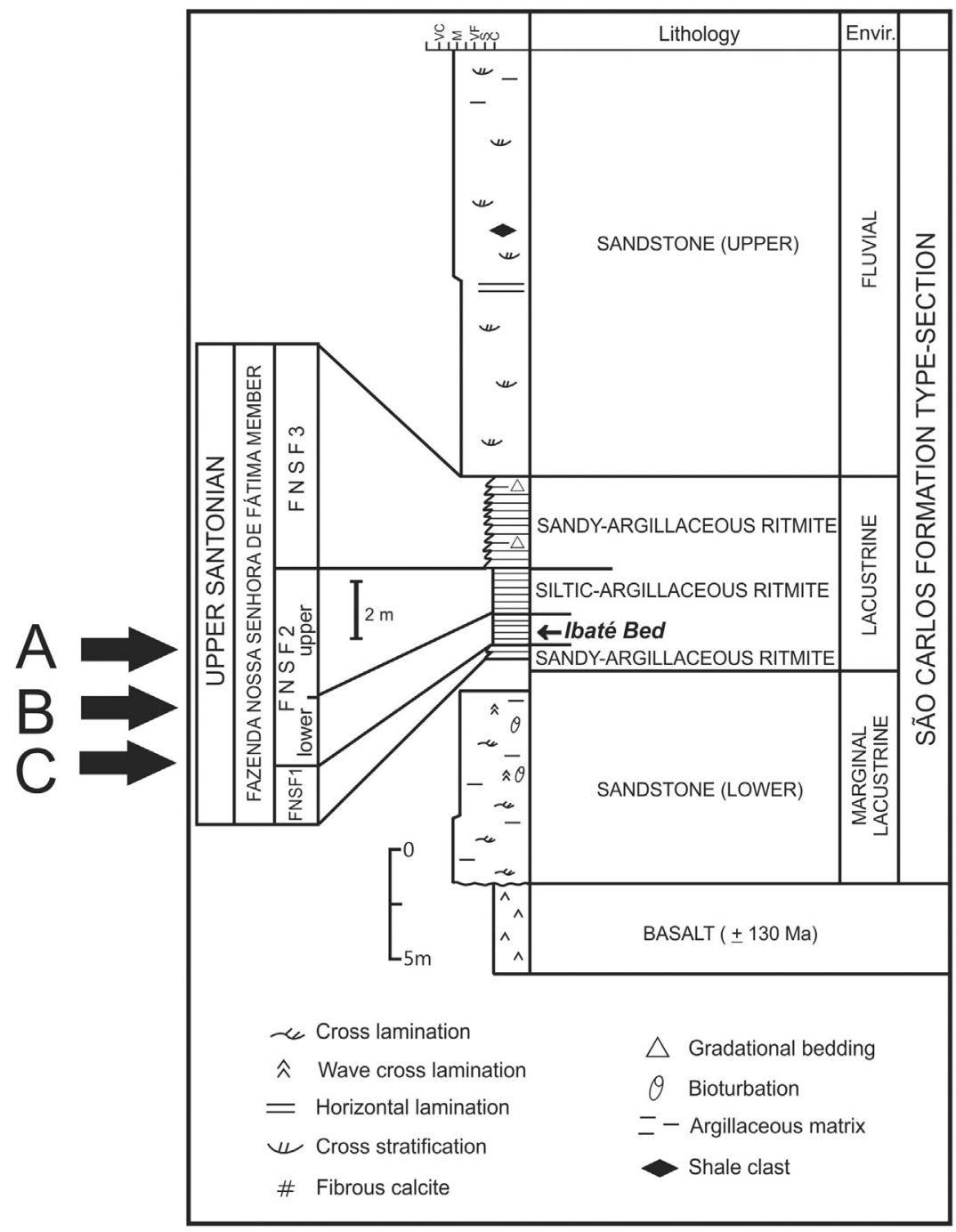

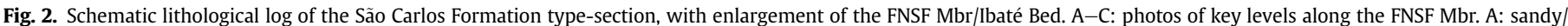

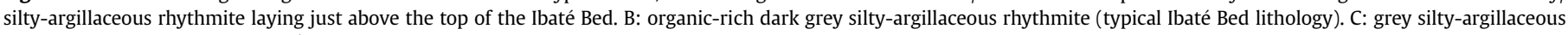
rhythmite at the base of the Ibaté Bed (modified from Castro et al., 2002).

especially well preserved palynomorphs - UNESP-5 and UNESP-6 were documented by scanning electron microscopy (SEM) at Petrobras Research Center (Rio de Janeiro, Brazil), using the Zeiss EVO 40 apparatus. Additional SEM images for details of Anacolosidites eosenonicus and Tricolpites joelcastroi, two new pollen species, were obtained with a Zeiss EVO MA15, of the UNESPetro SEM Laboratory. All the palynological slides, including those with the new taxa, are deposited in the UNESP (São Paulo State University) collection, IGCEUNESPetro, in Rio Claro, Brazil, and are referred to as UNESP $\lambda$-BP. For the comparison of the assemblage with those of the distinct phytogeographic provinces, we have chosen papers that bring at least three common species and respective photomicrographs from the worldwide literature.

\section{Results}

\subsection{Palynological content}

The assemblages consist of spores (32 taxa), gymnosperm pollen grains ( 31 taxa), angiosperm pollen grains (64 taxa), microalgal remains ( 9 taxa), fungal remains (one taxon) and phytoclasts (one seed cuticle type, 11 astrosclereid morphotypes and 3 trichome morphotypes), as illustrated in Figs. 3-14 and listed in the
Appendix (in Supplementary material). The quantitative data (absolute counts) of these palynomorphs are given in Supplementary Table A.

Regali et al. (1974a) stated that Brazilian palynofloras were dominated by gymnosperms until the Turonian, and the diversity of angiosperms increased significantly in the interval ConiacianSantonian. The palynoflora studied here is compatible with that assumption, presenting angiosperm diversity higher than that of gymnosperm, although quantitatively gymnosperm pollen was still dominant.

\subsection{Systematic paleontology}

Most taxa noted here have already been described in previous publications (see Appendix - in Supplementary material). However, we present 7 new species (two pollen grains, one microphytoplankton element and four phytoclasts). Among the phytoclasts, two new genera are proposed: Stellatia (fossil astrosclereids) and Trichomites (fossil trichomes).

Pollen [Pollenites, R. Potonié 1931]

Genus Anacolosidites Cookson \& Pike, 1954, emend. Potonié, 1960 Type species: Anacolosidites luteoides Cookson \& Pike, 1954 

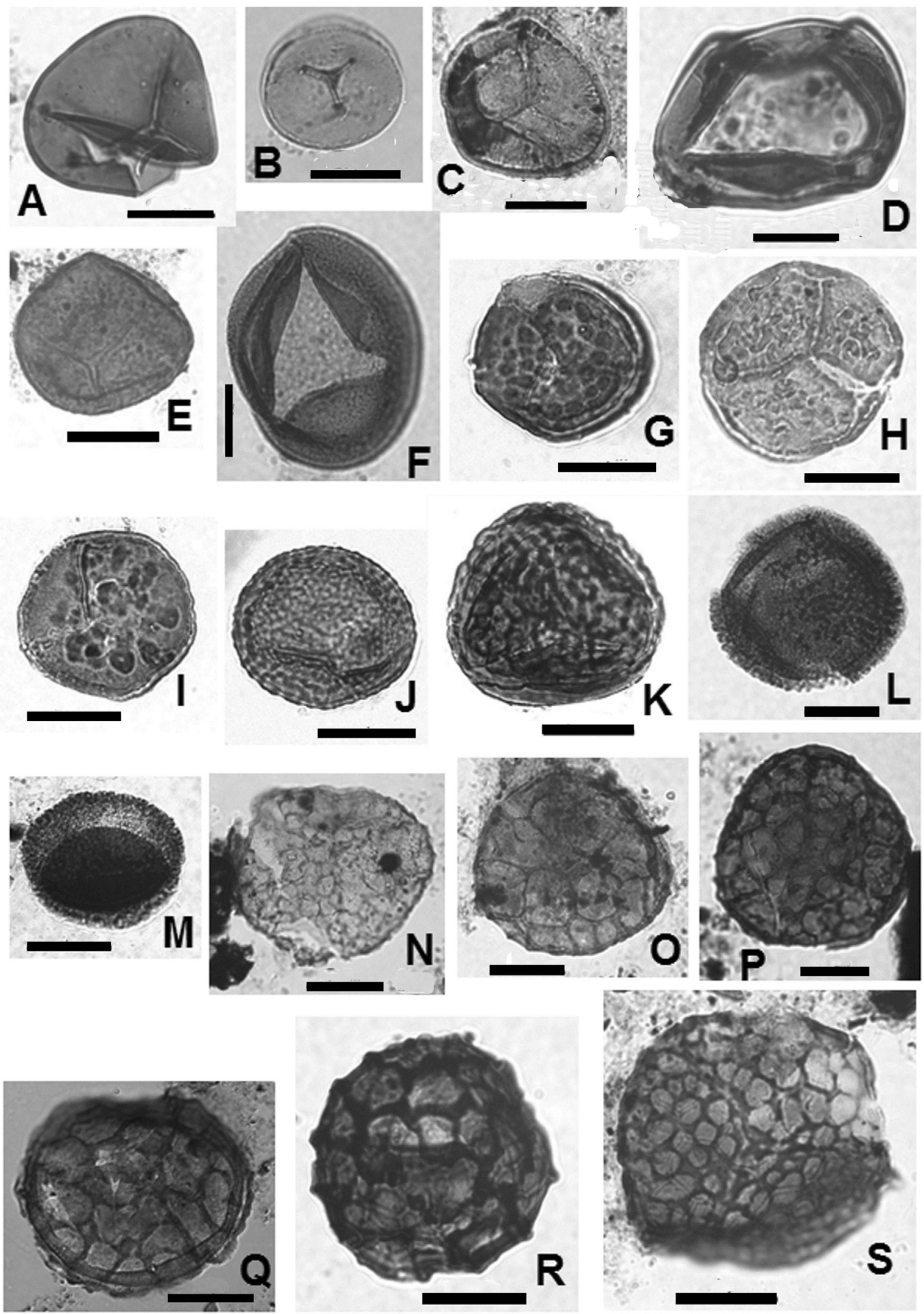

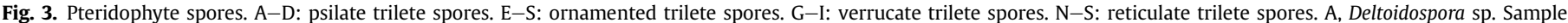

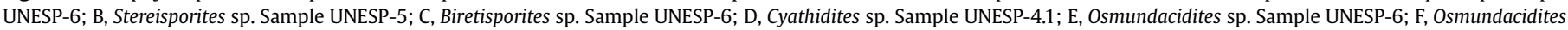

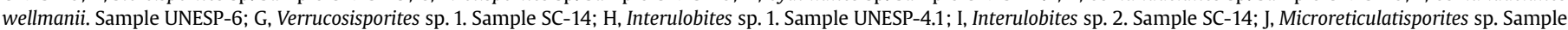

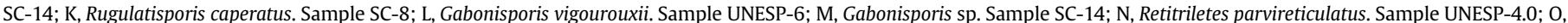

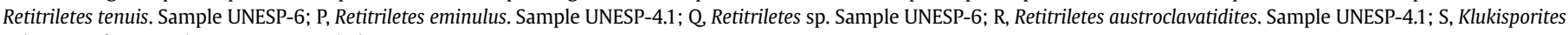
sphaerogoufus. Sample UNESP-4.1. Scale bars $=20 \mu \mathrm{m}$. 

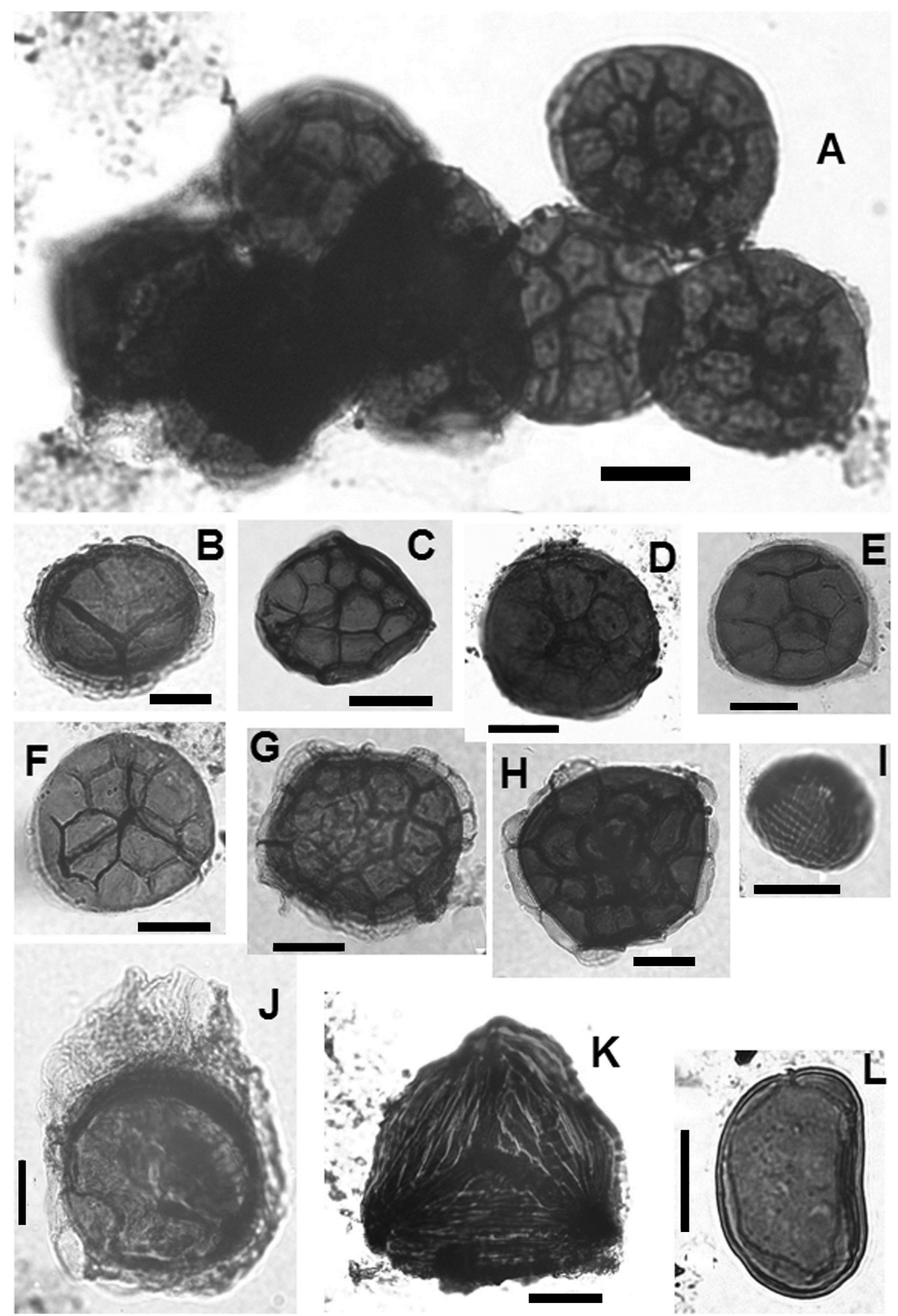

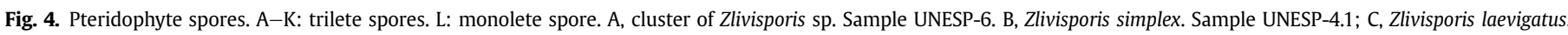

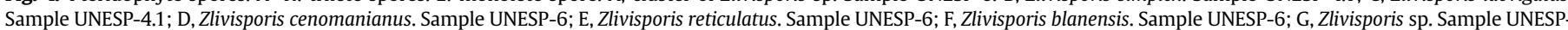

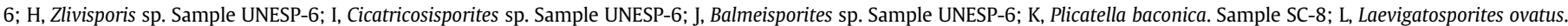
Sample SC-14. Scale bars $=20 \mu \mathrm{m}$. 

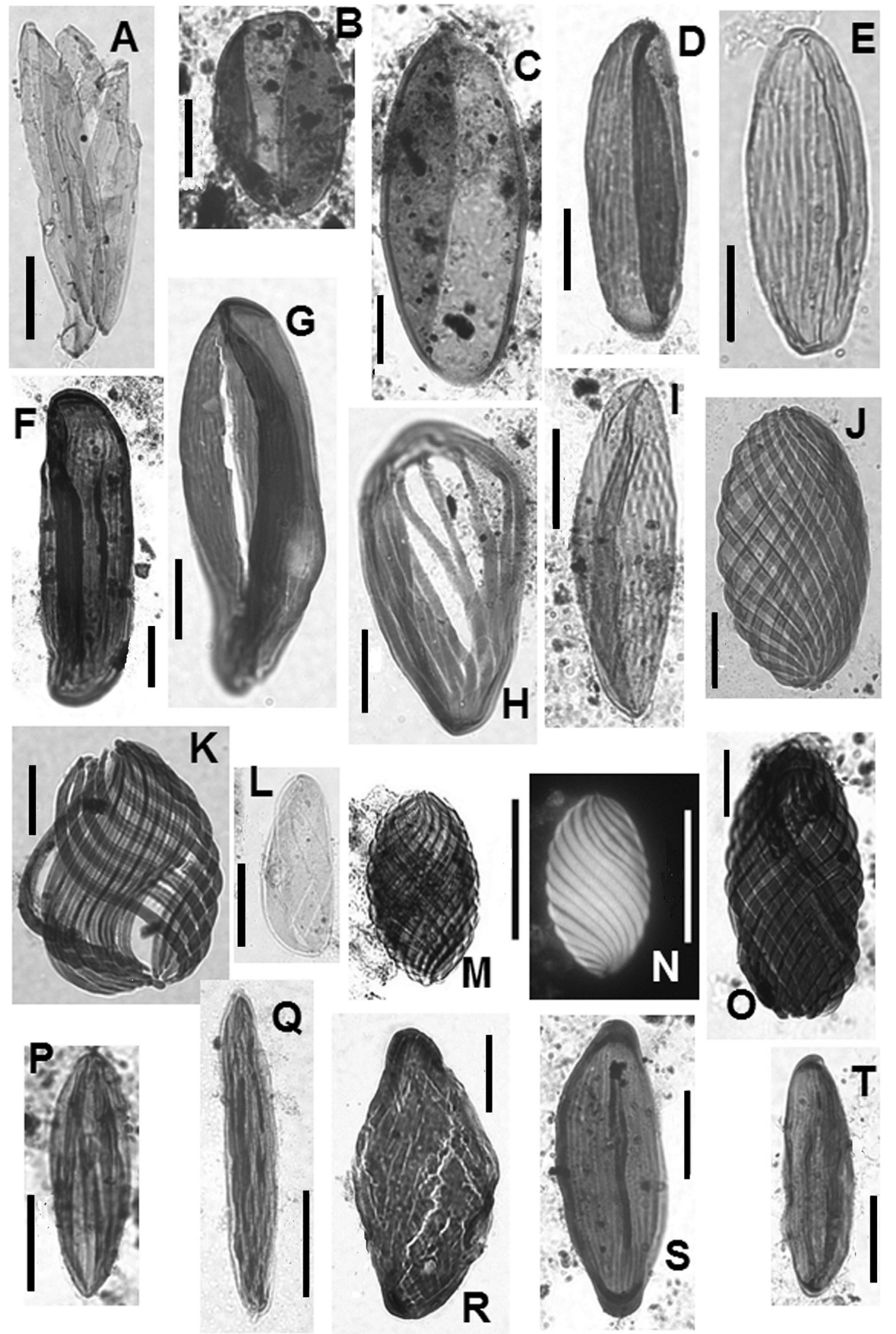

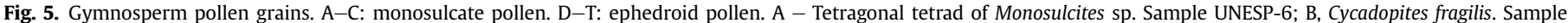

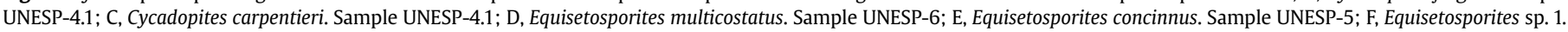

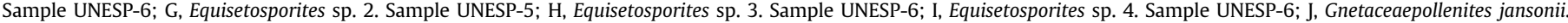

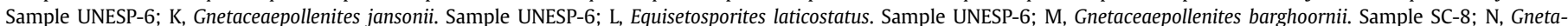

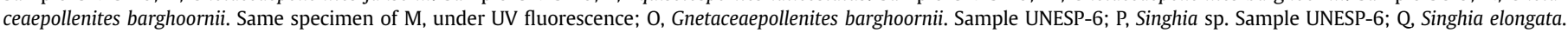

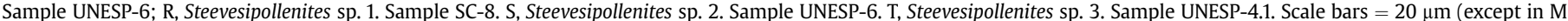
and $\mathrm{N}$ having $50 \mu \mathrm{m}$ ). 

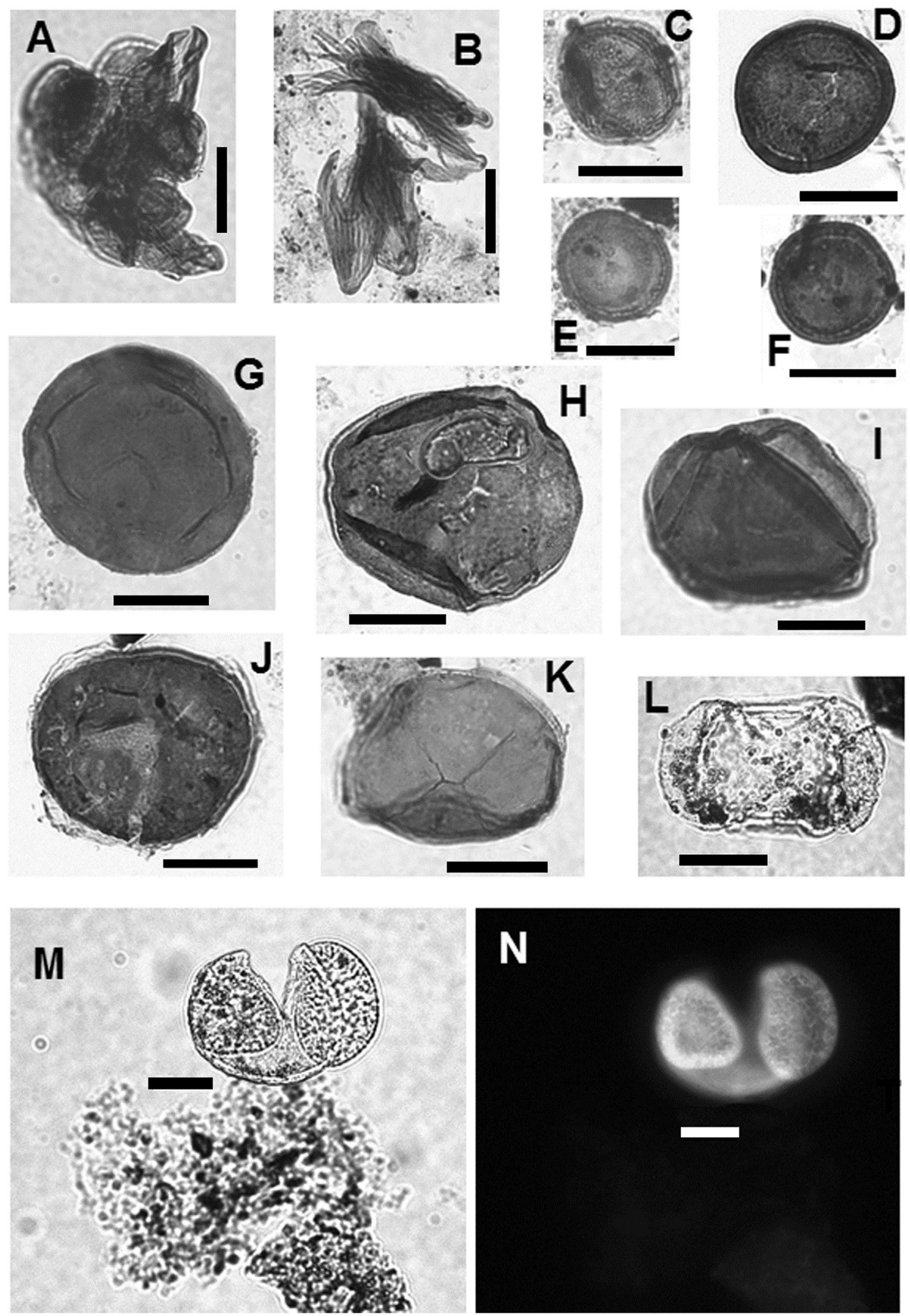

Fig. 6. Gymnosperm pollen. A-B: ephedroid pollen. C-F: Classopollis-type pollen. G-K: inaperturate pollen. L-N: bisaccite pollen. A, Cluster of Equisetosporites sp. Sample UNESP4.1; B, Disaggregated tetrad of Steevesipollenites sp. Sample UNESP-4.1; C, Classopollis sp. Sample UNESP-4.1; D, Classopollis sp. Sample SC-14; E, Classopollis classoides. Sample UNESP-6; F, Classopollis sp. Sample UNESP-4.1; G, Inaperturopollenites sp. Sample UNESP-6; H, Callialasporites aff. trilobatus. Sample SC-14; I, Callialasporites trilobatus. Sample UNESP-4.1; J, Inaperturopollenites simplex. Sample SC-14; K, Inaperturopollenites cf. simplex. Sample UNESP-4.0; L, Rugubivesiculites reductus. Sample SC-13; M, Podocarpidites sp. and amorphous organic matter. Sample SC-8; N, Podocarpidites sp. Same microscopic field of M under UV fluorescence. Note that this amorphous organic matter is devoid of fluorescence. Scale bars $=20 \mu \mathrm{m}$. 

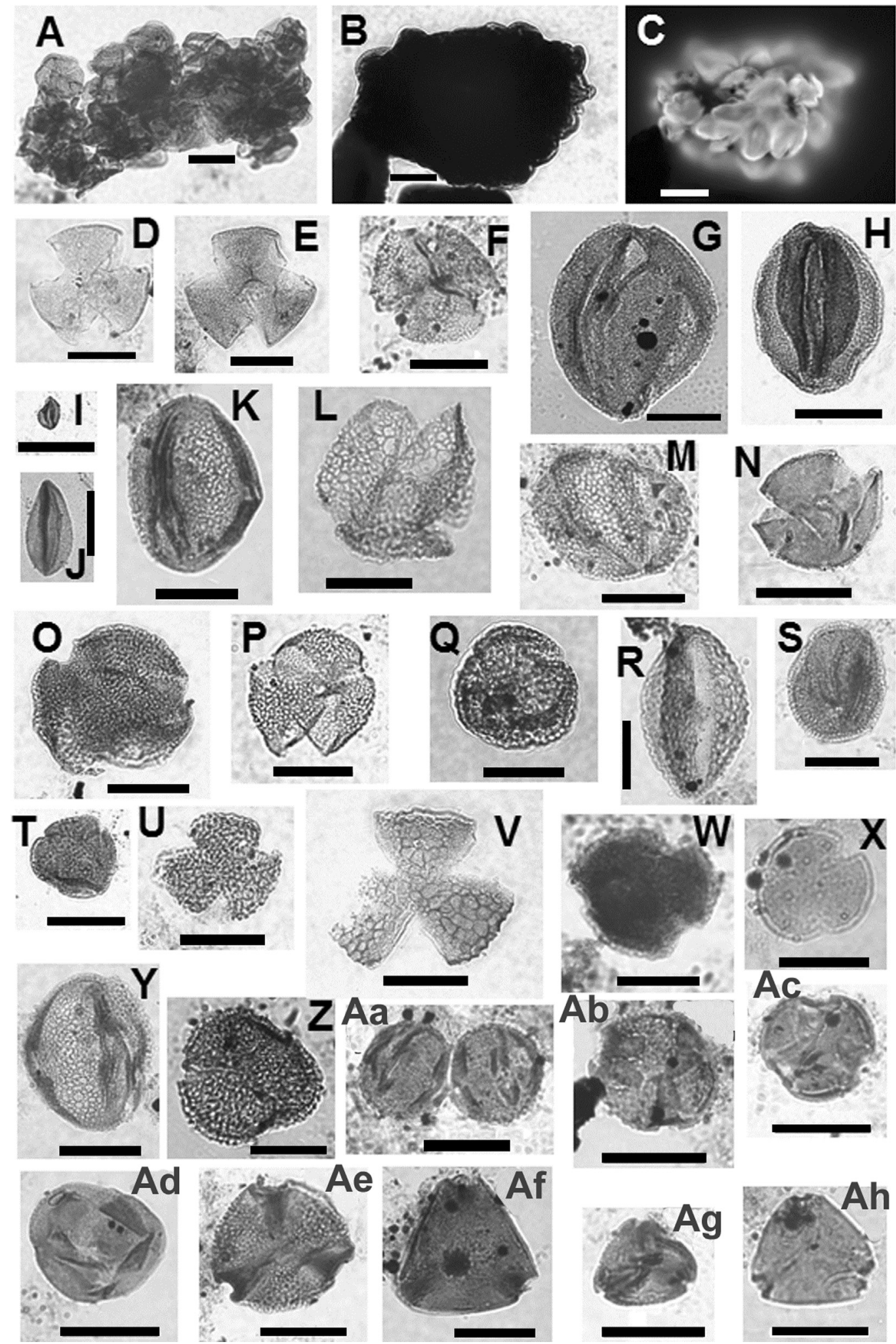

Fig. 7. Angiosperm pollen. A-W: tricolpate pollen grains. X - Z; 1-8: tricolporate pollen grains. A, Cluster of Tricolpites minutus. Sample UNESP-6; B, Cluster of Cupuliferoidaepollenites parvulus. Sample SC-8; C, Same microscopic field of B under UV fluorescence; D, Tricolpites sagax. Sample UNESP-4.1; E, Tricolpites joelcastroi. Sample SC-8; F, Tricolpites vulgaris. Sample UNESP-4.1; G, Foveotricolpites gigantoreticulatus. Sample UNESP-6; H, Foveotricolpites tienabaensis. Sample SC-14; I, Tricolpites sp. Sample SC-14; J, Faxinoipollenites sp. Sample UNESP-6; K, Rhoipites sp. Sample UNESP-5; L, Satishia nigra. Sample UNESP-6; M, Retitricolpites virgeus. Sample UNESP-4.1; N, Baculatitricolpites manifestus. Sample SC-14; O, Foveotricolpites giganteus. Sample SC-14; P, Retitricolpites gageonnetti. Sample SC-14; Q, Hammenia triangula. Sample SC-8; R, Rousea patagonica. Sample UNESP-6; S, Retitrescolpites saturnum. Sample UNESP-4.1; T, Hammenia fredericksburgensis. Sample SC-14; U, Albertipollenites anguloluminosus. Sample SC-14; V, Albertipollenites sp. Sample SC-14; W, Tricolpites foveolatus. Sample UNESP-6; X, Nyssapollenites squamosus. Sample UNESP-6; Y, Retitricolporites sp. Sample UNESP-6; Z, Retitricolporites salardii. Sample UNESP-6; Aa, Tricolporopollenites foveotectatus. Sample UNESP-6; Ab, Retitricolporites fekelensis. Sample UNESP-5; Ac, Tricolporopollenites micromurus. Sample UNESP-6; Ad, Tricolporopollenites subobscurus. Sample UNESP-6; Ae, Margocolporites mandjicus. Sample UNESP-4.1; Af, Tricolporopollenites sp. Sample UNESP-4.1; Ag, Tricolporopollenites aliquantulus. Sample UNESP-4.1; Ah, Sohlipollis sp. Sample UNESP-4.1. Scale bars $=20 \mu \mathrm{m}$. 

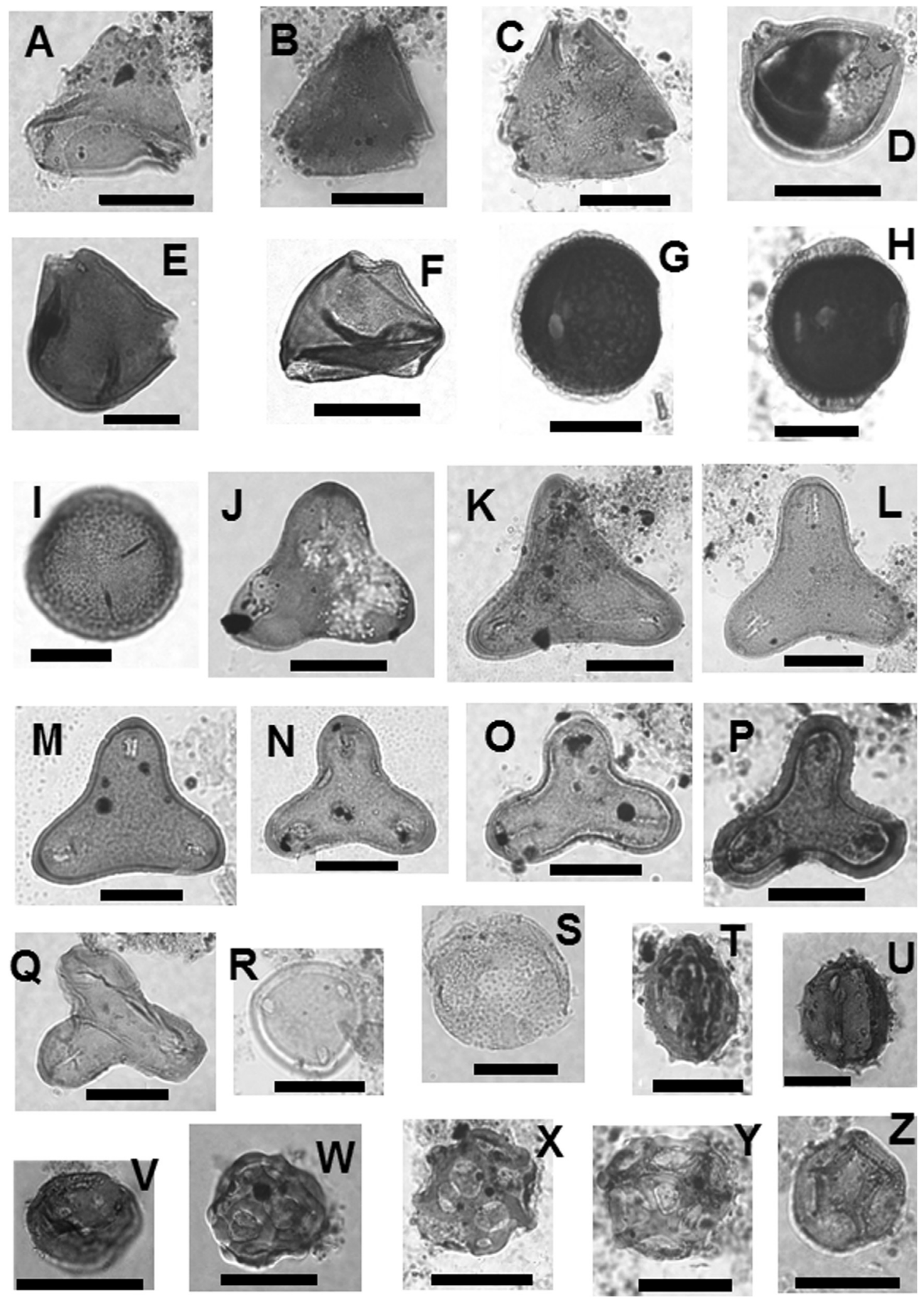

Fig. 8. Angiosperm pollen. A - C; G-H: tricolporate pollen grains. D - F; I-R: pollen grains belonging to group Victorisporis/Anacolosidites. S-Z: pollen grains with other aperture types. A, Tricolporopollenites sp. Sample UNESP-4.0; B, Lewalanipollis cf. senectus. Sample UNESP-6; C, Holkopollenites chemardensis. Sample UNESP-4.1; D, Trudopollis sp. Sample UNESP-4.0; E, Victorisporis sp. Sample UNESP-4.1; F, Lewalanipollis senectus. Sample SC-14; G, Retitricolporites belmontensis. Sample UNESP-6; H, Retitricolporites belmontensis. Sample UNESP-6; I, Constantinisporis jacquei. Sample UNESP-4.1; J, Anacolosidites sp. Sample UNESP-4.1; K, Anacolosidites eosenonicus. Sample UNESP-6; L, Anacolosidites eosenonicus. Paratype. Sample UNESP-5; M, Anacolosidites eosenonicus. Holotype. Sample UNESP-6; N, Anacolosidites eosenonicus. Sample UNESP-6; O, Anacolosidites eosenonicus. Sample UNESP4.1; P, Anacolosidites eosenonicus. Sample UNESP-6; Q Anacolosidites eosenonicus. Sample UNESP-4.0; R, Victorisporis robertii. Sample UNESP-4.0; S, Cretacaeiporites scabratus. Sample UNESP-5; T, Hexaporotricolpites emelianovi. Sample UNESP-4.1; U, Hexaporotricolpites emelianovi. Sample UNESP-4; V, Psilaperiporites sp. Sample UNESP-6; W, Confossia vulgaris. Sample UNESP-4.1; X, Confossia vulgaris. Sample UNESP-4.1; Y, Confossia vulgaris. Sample UNESP-6; Z, Cretacaeiporites polygonalis. Sample UNESP-6; Scale bars = 20 mm. 

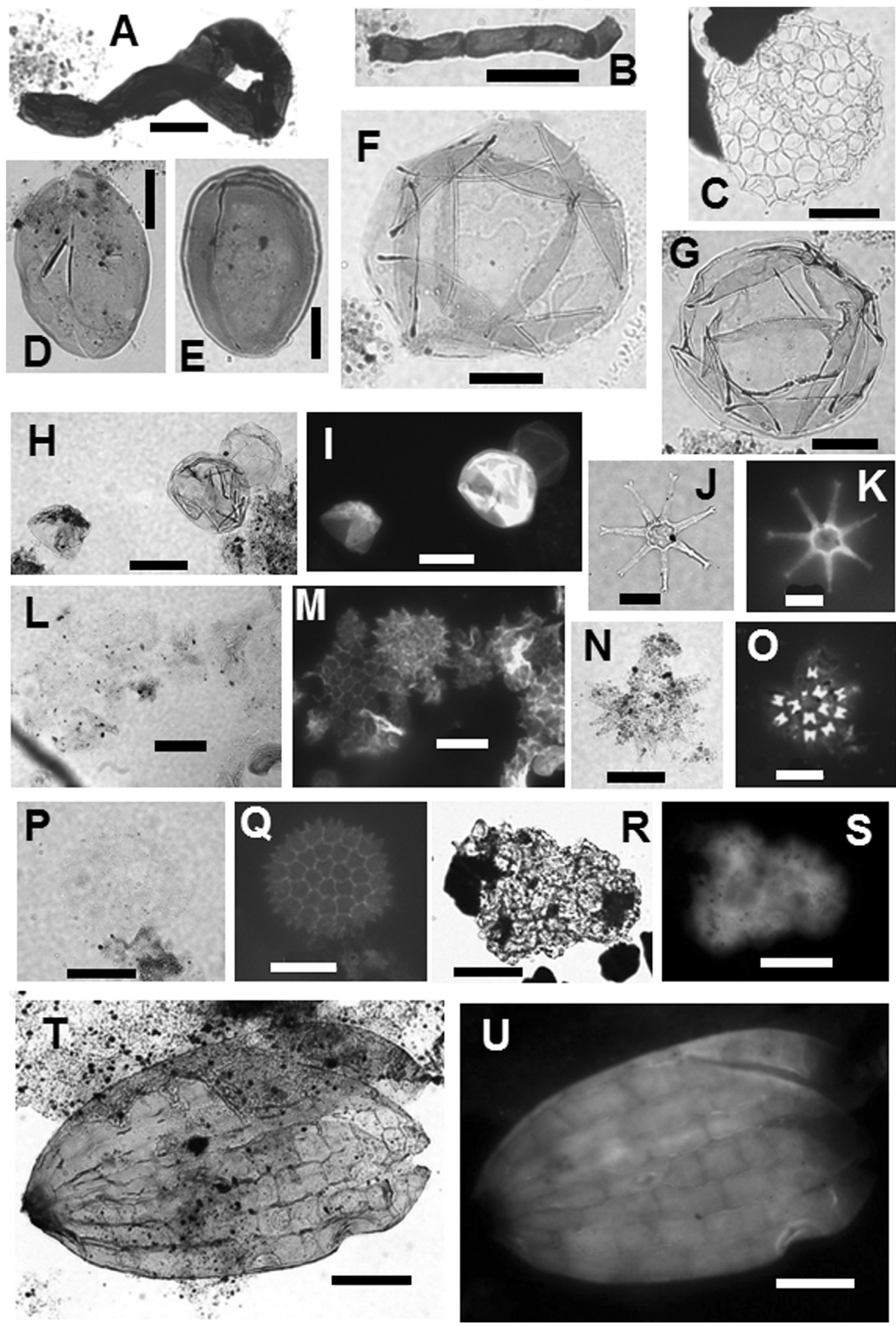

Fig. 9. Miscellaneous. A-B: fungal remains. C: Incertae sedis. D-S: algal remains. T-U: seed remain. A, Fungal remain (hyphae). Sample UNESP-4.1; B, Fungal remain (hyphae). Sample UNESP-6; C, Schizosporis aff. reticulatus. Sample UNESP-4.1; D, Brazilea parva. Sample UNESP-6; E, Brazilea parva. Sample UNESP-4.1; F, Leiosphaeridia ibateensis. Sample UNESP-5; G, Leiosphaeridia ibateensis. Sample UNESP-5; H, Microscopic field with 3 specimens of L. ibateensis. Sample SC-8; I, Same field of H under UV fluorescence; J. Staurastrum sp. Sample SC-11; K, Same field of J under UV fluorescence; L, Microscopic field with Pediastrum spp. Sample SC-14; M, Same field of L under UV fluorescence; N, Pediastrum aff. mustersii. Sample SC-13; O, same specimen of N under UV fluorescence; P, Pediastrum boryanum. Sample UNESP-4.0; Q Same specimen of P under UV fluorescence; R, Botryococcus sp. Sample SC-15; S, Same specimen of R under UV fluorescence; T, Spermatites sp. Sample SC-7A; U, Same specimen of T under UV fluorescence. Scale bars $=20 \mu \mathrm{m}$ (except in H, I, L, M, P, Q R, S, T and U having $50 \mu \mathrm{m}$ ) 
Generic description: besides the original diagnosis (Cookson \& Pike, 1954) and the emended diagnosis (Potonié, 1960), we adopted the description for Anacolosidites proposed by Malécot \& Lobreau-Callen (2005, p. 318) because of its more complete characterization.

\section{Anacolosidites eosenonicus sp. nov.}

Figs. $8 \mathrm{~K}-\mathrm{Q}$ and $14 \mathrm{C}-\mathrm{F}$

1977 Cf. Accuratipollis sp. in Ioannides \& Colin, plate 4, figures 20-21 (p. 897).

2002 Anacolosidites? sp. A in Castro et al. (p. 356).

2006 Cf. Anacolosidites sp. in Arai et al., figure 2C (p. 128).

2016 Anacolosidites sp. A in Premaor, figure II.5S (v. 2, p. 14).

Holotype: Slide UNESP-6, C49 (Fig. 8M).

Paratype: Slide UNESP-5, C44/3 (Fig. 8L).

Type locality: Fazenda Nossa Senhora de Fátima (Ibaté municipality, São Paulo State, Brazil. UTM 237577.6 km, 197.75 km).

Type stratum: São Carlos Formation, FNSF Mbr, Ibaté Bed, upper Santonian.

Description: Isopolar pollen with radial symmetry; pollen outline triquete (strongly triangular concave) in polar view and peroblate in equatorial view; grain hexaporate (tri-diporate) with three pairs of elongate pores parallel to radius, located near the equator (about $1 / 6$ of the equatorial diameter in from the perimeter of the grain); exine thickness normally uniform, but sometimes convex parts can present slightly thicker exine; ornamentation psilate to scabrate under the optical microscope, but observations under SEM reveal that it is characterized by tightly packed granules (see Fig. 14C-F).

Dimensions: holotype - total diameter $=47 \mu \mathrm{m}$; paratype - total diameter $=46 \mu \mathrm{m}$; diameter range $=32$ (37) $47 \mu \mathrm{m}$ (24 specimens); size of granules $=0.6-0.9 \mu \mathrm{m}$; exine thickness $=2-3 \mu \mathrm{m}$.

Derivation of name: from "Eosenonian" (early Senonian) that corresponds approximately to the interval Coniacian-Santonian that is the stratigraphic range of this species.

Remarks: Anacolosidites eosenonicus must be the oldest Anacolosidites species reported worldwide. Among the species mentioned in the inventory by Malécot \& Lobreau-Callen (2005), Anacolosidites striatus Wiggins, reported from the Campanian of Alaska, was the oldest one. All other Anacolosidites species occur in the Maastrichtian-Neogene interval.

Comparison: A. striatus and A. eosenonicus are only two known Anacolosidites species having strongly triangular concave outline. A. striatus differs from A. eosenonicus in having a distinct striate ornamentation.

Botanical affinity: possibly Loranthaceae, because Anacolosidites striatus that is morphologically the closest species of $A$. eosenonicus was considered as possible Loranthaceae by Malécot \& LobreauCallen (2005).

Genus Tricolpites Cookson, 1947 ex Couper, 1953

Type species: Tricolpites reticulatus Cookson, 1947 ex Couper, 1953

Tricolpites joelcastroi sp. nov.

Figs. 7E, 13H-L

Holotype: Slide SC-8, Q57 (Fig. 7E).

Type locality: Fazenda Nossa Senhora de Fátima (Ibaté municipality, São Paulo State, Brazil. UTM 237577.6 km, 197.75 km).

Type stratum: São Carlos Formation, FNSF Mbr, Ibaté Bed, upper Santonian.

Description: Isopolar tricolpate pollen with radial symmetry; amb subtriangular in polar view and oblate to prolate spheroidal in e-

q- uatorial view; three conspicuous colpi; exine thickness uniform; ornamentation scabrate seen under SEM to be granulate with granules distributed uniformly (Fig. 13I, K).

Dimensions: holotype - total diameter $=44 \mu \mathrm{m}$; granule diameter $=0.2-0.5 \mu \mathrm{m}$; total diameter range $=19(26) 44 \mu \mathrm{m}(10$ specimens); exine thickness $=0.5-1.0 \mu \mathrm{m}$; density of granule $=4-6$ granules per one square micrometer.

Derivation of name: in honour to Joel Carneiro de Castro, recognized Brazilian sedimentologist and stratigrapher and one of authors who established the São Carlos Formation.

Remarks: this species is the only tricolpate pollen having a conspicuous granulate ornamentation in the studied assemblage. Tricolpites joelcastroi resembles Verrutricolpites sp. illustrated by Vallati (2006, p. 89, fig. 6C), but differs in having finer verrucae (microverrucae).

Botanical affinity: indeterminate dicotyledon (Class Magnoliopsida).

Acritarchs [Acritarcha, Evitt 1963]

Genus Leiosphaeridia Eisenack, 1958

Type species: Leiosphaeridia baltica Eisenack, 1958

Leiosphaeridia ibateensis sp. nov.

Figs. 9F-G, 14I-J

Holotype: Slide UNESP-5, M63 (Fig. 9F).

Paratype: Slide SC-8, C57/1 (Fig. 9G).

Type locality: Fazenda Nossa Senhora de Fátima (Ibaté municipality, São Paulo State, Brazil. UTM 237577.6 km, 197.75 km).

Type stratum: São Carlos Formation, FNSF Mbr, Ibaté Bed, upper Santonian.

Description: organic-walled spherical vesicle; pylome absent; smooth thin wall which is conspicuously folded; high fluorescence under UV.

Dimensions: holotype - total diameter $=78 \mu \mathrm{m}$; paratype - total diameter $=72 \mu \mathrm{m}$; total diameter range $=60(71) 80 \mu \mathrm{m}(22$ specimens); vesicle wall thickness $=$ ca. $1 \mu \mathrm{m}$.

Derivation of name: from Ibaté municipality, where the São Carlos Formation type-section is located.

Botanical affinity: probably a prasinophyte. The prasinophycean affinity is compatible with its high fluorescence (Fig. 9I). This is also compatible with the point of view of Wall (1965) and Guy-Ohlson (1996).

Comparison: Among fresh-water Leiosphaeridia-type vesicles, Leiosphaeridia ibateensis is the biggest species according the following total diameter comparisons: Leiosphaeridia sp. B (in Volkheimer et al., 1977): 14-25 $\mu \mathrm{m}$; Leiosphaeridia menendezii Volkheimer et al., 1977: 21-32 $\mu \mathrm{m}$; Leiosphaeridia dellapei Volkheimer et al., 1977: 14-25 $\mu \mathrm{m}$; Leiosphaeridia sp. (in Prámparo \& Volkheimer, 2002): 25-35 $\mu \mathrm{m}$; Leiosphaeridia-type 1 (in Narváez et al., 2014): 32-46 $\mu \mathrm{m}$.

Phytoclasts

Stellatia gen. nov.

Diagnosis: fossil solid star-shaped organic particle having thick and opaque wall and which appears in palynological residues. Type species: Stellatia furcata (Duarte \& Arai) comb. nov. Derivation of name: latin Stella - meaning star; star-shaped. Remarks: The genus Stellatia is proposed herein to designate fossil remains derived from astrosclereids of vascular plants in substitution to 'fossil sclereids' proposed by Duarte \& Arai (2010) as a new category of palynomorphs sensu lato.

Stellatia furcata (Duarte \& Arai) comb. nov. 

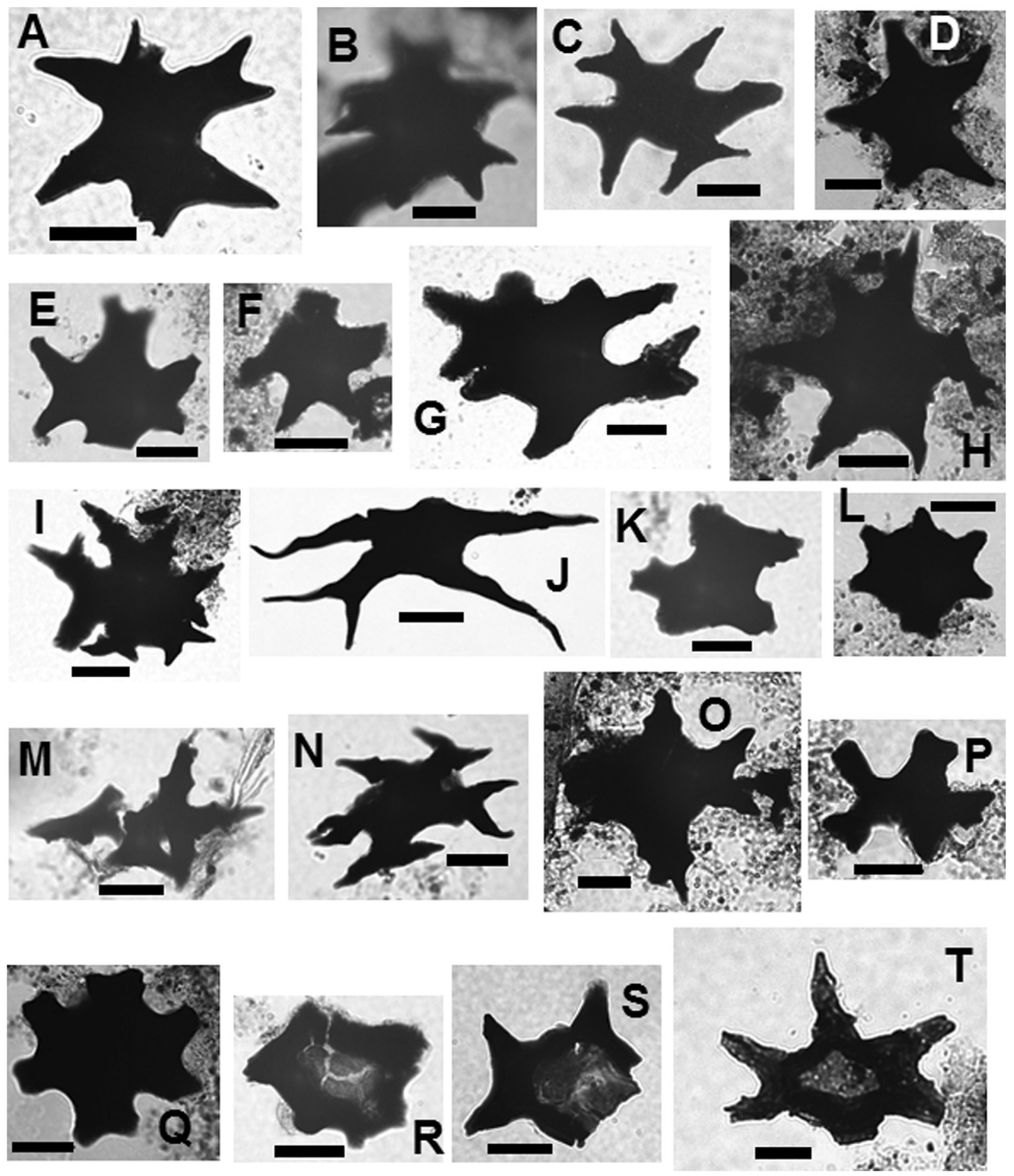

Fig. 10. Stellatia spp. (fossil astrosclereids). A, Stellatia furcata. Paratype. Sample SC-8; B, Stellatia furcata. Sample UNESP-6; C, Stellatia furcata. Holotype. Sample UNESP-4.1; D, Stellatia simplex. Holotype. Sample UNESP-6; E, Stellatia furcata. Sample UNESP-5; F, Stellatia simplex. Paratype. Sample UNESP-5; G, Stellatia furcata. Sample SC-8; H, Stellatia spinosa. Sample UNESP-4.1; I, Stellatia aculeata. Sample SC-8; J, Stellatia elongata. Sample UNESP-6; K, Stellatia spinosa. Sample UNESP-5; L, Stellatia callosa. Sample UNESP-6; M, Stellatia spinosa. Sample UNESP-6; N, Stellatia furcata. Sample UNESP-4.1; O, Stellatia spinosa. Sample SC-8; P, Stellatia rotunda-truncata. Sample SC-8; Q Stellatia rotunda. Sample UNESP-6; R, Stellatia brevis-tenuis. Sample UNESP-6; S, Stellatia tenuis. Sample SC-8; T, Stellatia tenuis-spinosa. Sample SC-8. Scale bars $=20 \mu \mathrm{m}$.

Figs. 10A, B, C, E, G, N and 14L

2010 'Esclerócito tipo ramificado' (forma furcata) Duarte \& Arai (Fig. 4F-I)

Holotype: Slide UNESP-4.1, O67 (Fig. 10C = fig. 4G in Arai \& Duarte, 2010).

Paratype: Slide SC-8, S42/3 (Fig. 10A).
Type locality: Fazenda Nossa Senhora de Fátima (Ibaté municipality, São Paulo State, Brazil UTM 237577.6 km, 197.75 km).

Type stratum: São Carlos Formation, FNSF Mbr, Ibaté Bed, upper Santonian.

Description: outline stellate; 5 main arms; 2 to 4 of them can present bifurcation, resulting to 7 to 10 terminal arms; opaque and dark-colored. 

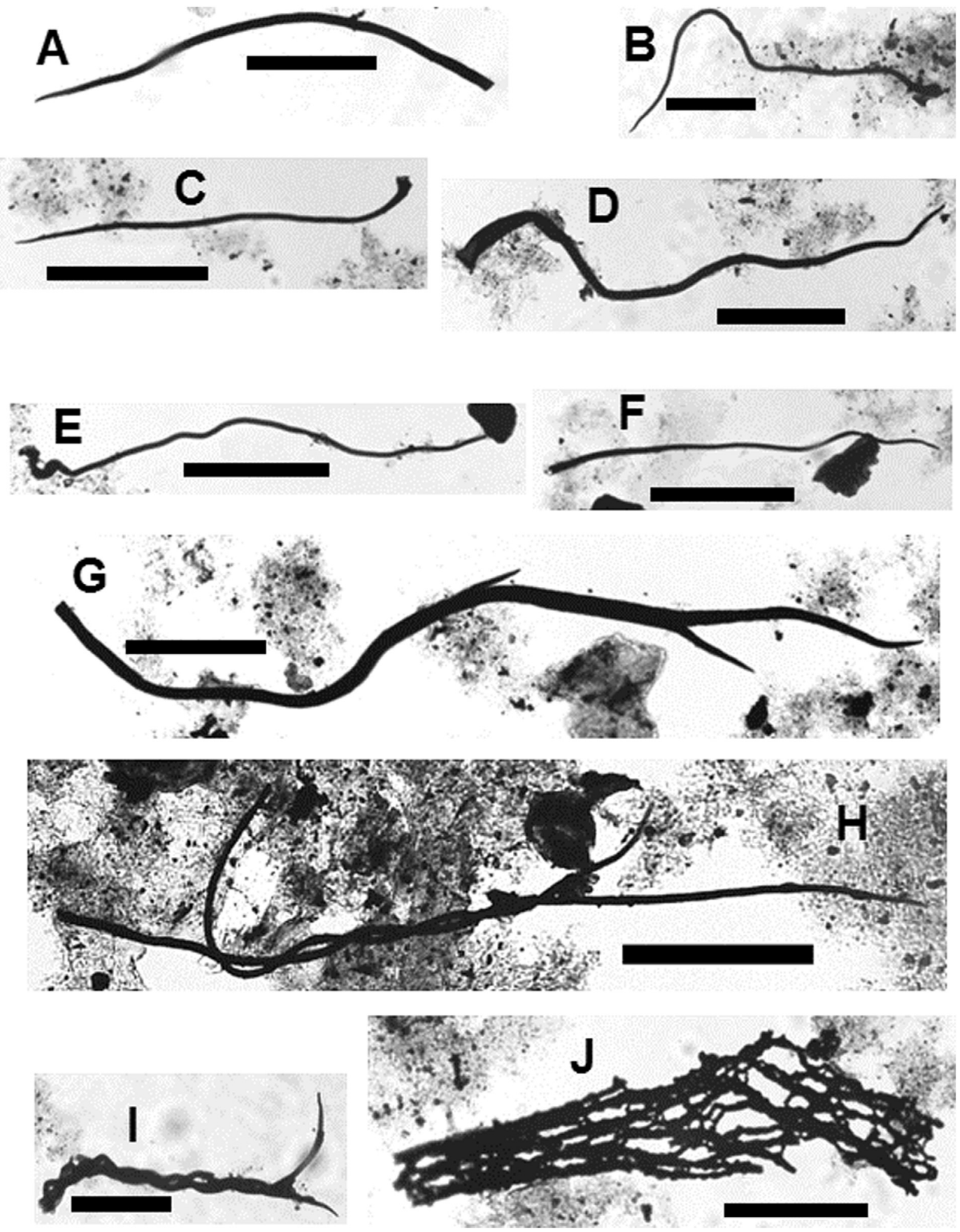

Fig. 11. Trichomites spp. (fossil trichomes) and woody organic matter. A, Trichomites simplex, thin recurved type. Sample UNESP-4.1; B, Trichomites simplex, thin sinuous type. Sample UNESP-4.0; C, Trichomites simplex, thin recurved type. Holotype. Sample UNESP-4.1; D, Trichomites simplex, thin sinuous type. Sample UNESP-4.1; E, Trichomites simplex, thin sinuous type. Sample UNESP-4.1; F, Trichomites simplex, thin sinuous type. Sample UNESP-6; G, Trichomites brevifurcatus. Holotype. Sample UNESP-4.1; H, Trichomites duplihelicoidus. Paratype. Sample SC-8; I, Trichomites duplihelicoidus. Holotype. Sample UNESP-6; J, Woody organic matter. Sample UNESP-6. Scale bars $=100 \mu \mathrm{m}$ (except in B and I with $50 \mu \mathrm{m}$ ). 

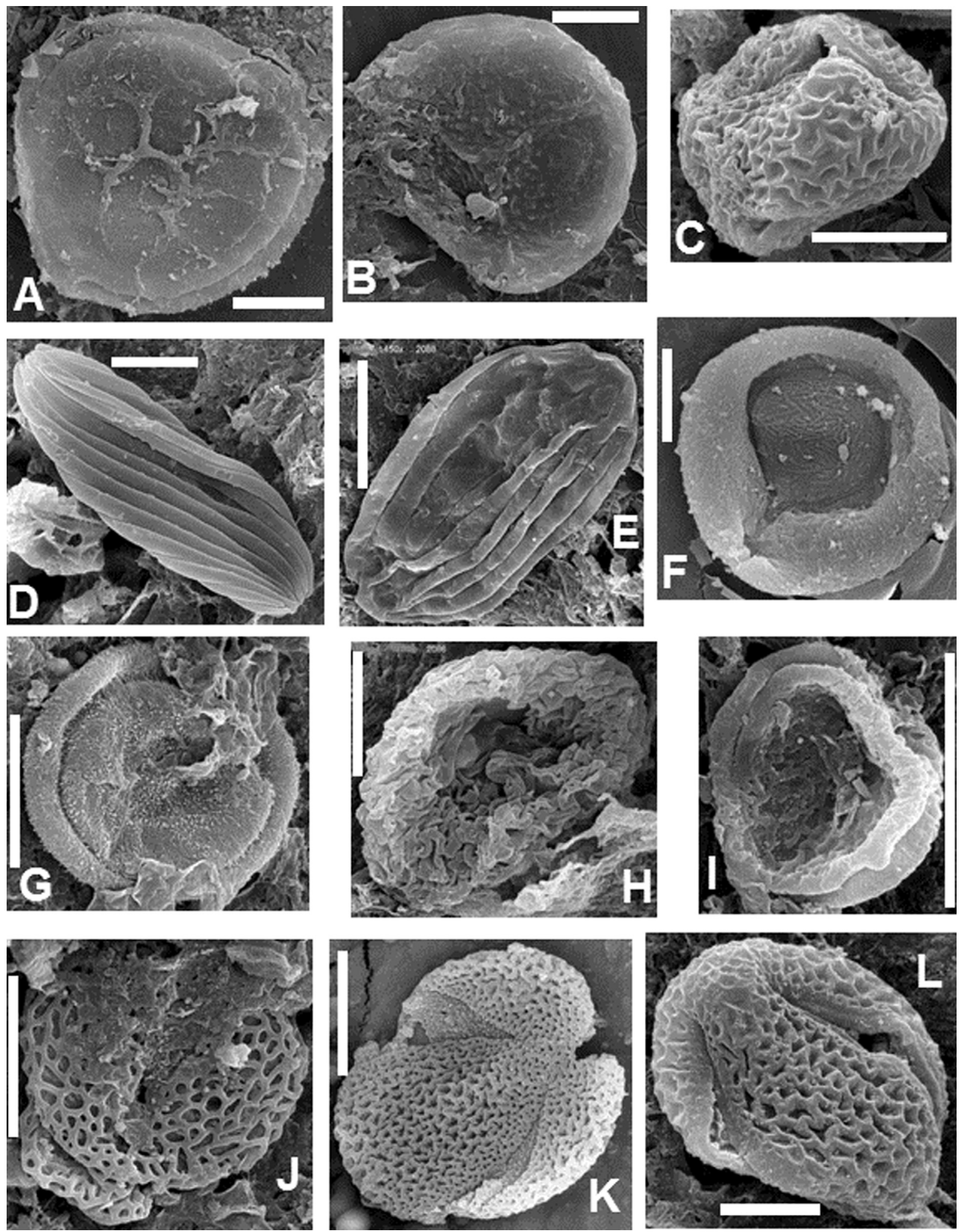

Fig. 12. SEM (Scanning Electron Microscope) photomicrographs. A, Zlivisporis reticulatus. Sample UNESP-6; B, Foraminisporis simiscalaris. Sample UNESP-6; C, Klukisporites sp. Sample UNESP-6; D, Gnetaceaepollenites barghoornii. Sample UNESP-6; E, Equisetosporites strigatus. Sample UNESP-5; F, Classopollis sp. Sample UNESP-6; G, Classopollis sp. Sample UNESP-6; H, Callialasporites ugensis. Sample UNESP-5; I, Proxapertites? sp. Sample UNESP-5; J, Retitricolpites sp. Sample UNESP-6; K, Dryadopollis sp. 1. Sample UNESP-6; L, Dryadopollis sp. 2. Sample UNESP-6. Scale bars $=20 \mu \mathrm{m}$ 

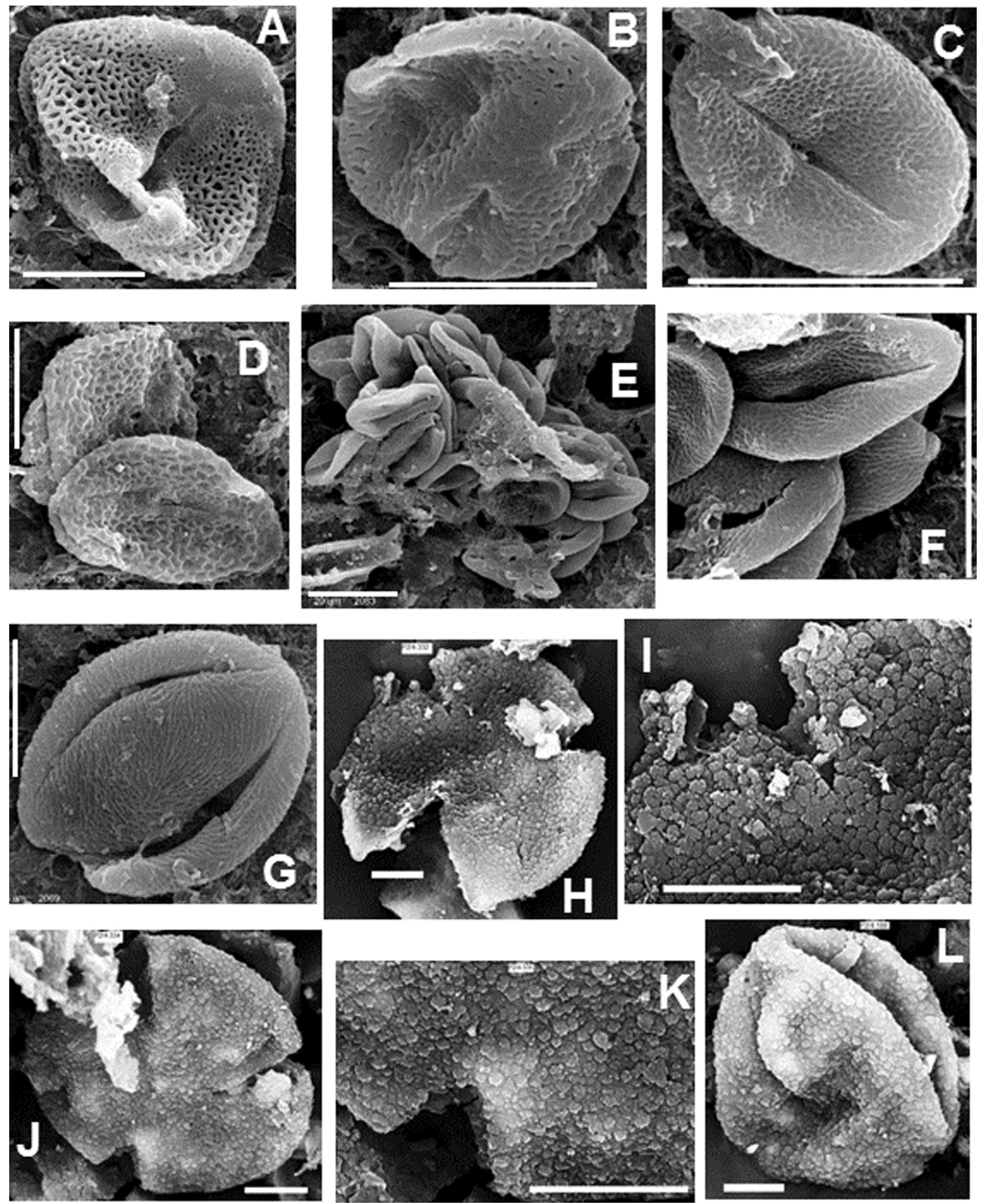

Fig. 13. SEM (Scanning Electron Microscope) photomicrographs. A, Retitricolporites sp. 1. Sample UNESP-6; B, Retitricolporites sp. 2. Sample UNESP-5; C, Retitricolporites sp. 3. Sample UNESP-5; D, Retitricolporites sp. 4. Sample UNESP-5; E, Cluster of Retitricolporites sp. 5. Sample UNESP-5; F, Retitricolporites sp. 5 (detail of lower right part of E); G, Striatopollis trochuensis. Sample UNESP-5; H, Tricolpites joelcastroi (polar view). Sample SC-8; I, T. joelcastroi (detail of upper part of H). Sample SC-8; J, T. joelcastroi (oblique polar view). Sample SC-8; K, T. joelcastroi (detail of lower part of J). Sample SC-8; L, T. joelcastroi (equatorial view). Sample SC-8. Scale bars = $20 \mu \mathrm{m}(\mathrm{A}-\mathrm{G}$ ); $5 \mu \mathrm{m}$ $(\mathrm{H}-\mathrm{L})$ 

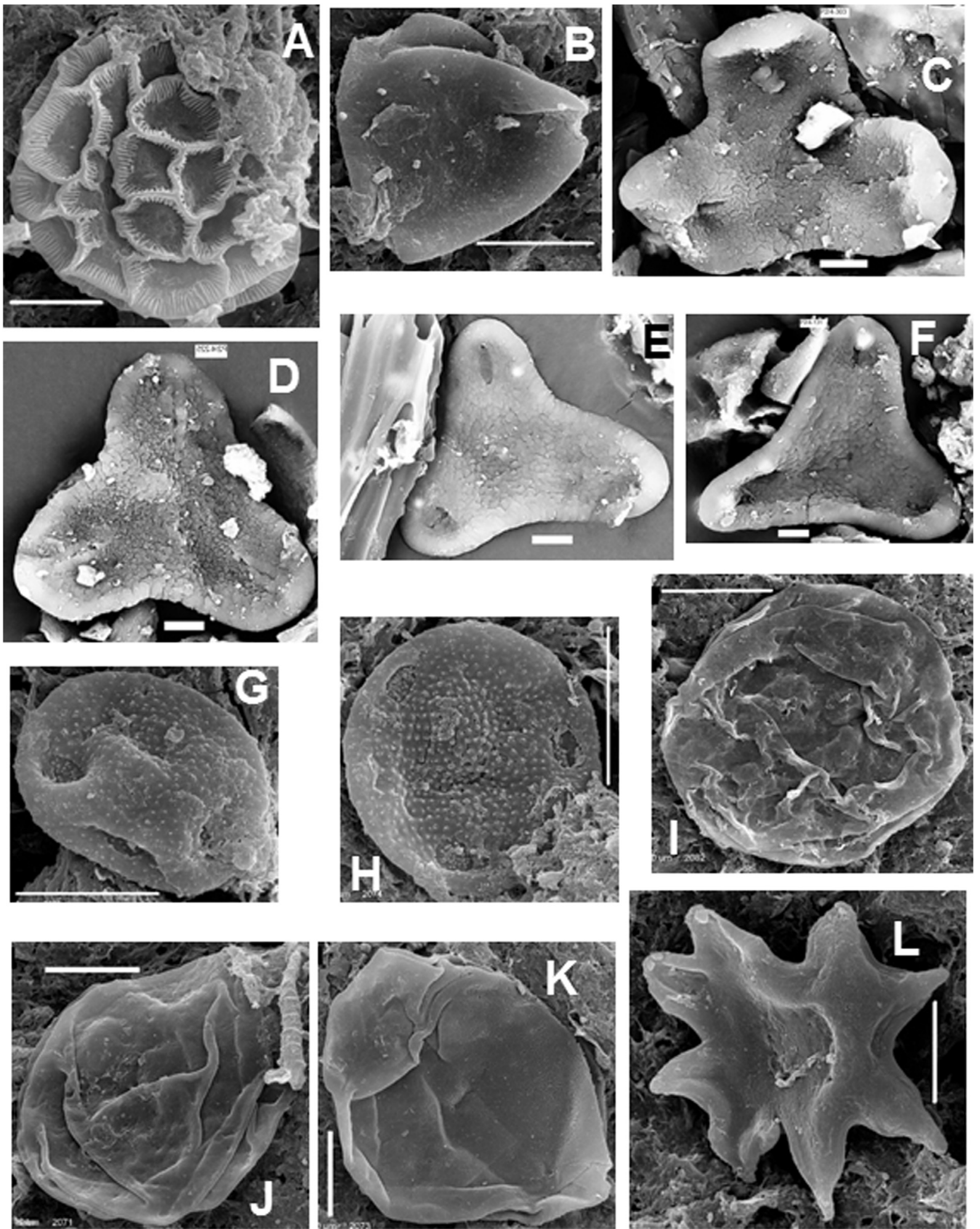

Fig. 14. SEM (Scanning Electron Microscope) photomicrographs. A, Retimonocolpites sp. Sample UNESP-6; B, Subtriporopollenites minutipori. Sample UNESP-6; C, Anacolosidites eosenonicus. Sample SC-14; D, A. eosenonicus. Sample SC-14; E, A. eosenonicus with charcoal (left). Sample SC-14; F, A. eosenonicus. Sample SC-14; G, Cretacaeiporites scabratus. Sample UNESP-5; H, Psilastephanoporites brasiliensis. Sample UNESP-5; I, Leiosphaeridia ibateensis. Sample UNESP-5; J, Leiosphaeridia ibateensis. Sample UNESP-5; K, Leiosphaeridia cf. ibateensis. Sample UNESP-5; L, Stellatia furcata. Sample UNESP-6. Scale bars $=20 \mu \mathrm{m}(\mathrm{A}, \mathrm{B}, \mathrm{G}-\mathrm{L}) ; 5 \mu \mathrm{m}(\mathrm{C}-\mathrm{F})$. 
Dimensions: holotype - total diameter $=90 \mu \mathrm{m}$; paratype - total diameter $=68 \mu \mathrm{m}$; total diameter range 64 (76) $99 \mu \mathrm{m}(7$ specimens).

Derivation of name: latin furcata - meaning branched.

Botanical affinity: according to Duarte \& Arai (2010), Nuphar spp. (Nymphaeaceae) produce very similar astrosclereids.

Trichomites gen. nov.

Diagnosis: all fossil plant-origin structures related to trichomes, as described by Arai \& Duarte (2010).

Type species: Trichomites brevifurcatus sp. nov.

Derivation of name: Trichome + suffix '-ites'.

Remarks: The genus Trichomites is proposed herein to designate fossil remains derived from trichomes of vascular plants in substitution to "fossil trichomes" proposed by Arai \& Duarte (2010) as a new category of palynomorphs sensu lato.

\section{Trichomites brevifurcatus sp. nov.}

Fig. 11G

Holotype: Slide UNESP-4.1, M54 (Fig. 11G).

Type locality: Fazenda Nossa Senhora de Fátima (Ibaté municipality, São Paulo State, Brazil UTM 237577.6 km, 197.75 km).

Type stratum: São Carlos Formation, FNSF Mbr, Ibaté Bed, upper Santonian.

Description: dark colored hair-like fossil trichome with short spines in alternating positions.

Dimensions (holotype): total length $=660 \mu \mathrm{m}$; length of spines $=30-40 \mu \mathrm{m}$; range of total length $=620$ (650) $690 \mu \mathrm{m}(5$ specimens).

Derivation of name: latin brevifurcatus - meaning short branches. Botanical affinity: probably Campanulaceae. According to Batterman \& Lammers (2004), trichomes with alternating branches are found in Lobelioideae (Campanulaceae).

Trichomites duplihelicoidus sp. nov.

Fig. $11 \mathrm{H}, \mathrm{I}$

2010 'Tricoma duplo delgado trançado' in Arai \& Duarte, fig. 3L (p. 181).

Holotype: Slide UNESP-6, Y8 (Fig. 11I).

Paratype: Slide SC-8, Q47 (Fig. 11H).

Type locality: Fazenda Nossa Senhora de Fátima (Ibaté municipality, São Paulo State, Brazil UTM 237577.6 km, 197.75 km).

Type stratum: São Carlos Formation, FNSF Mbr, Ibaté Bed, upper Santonian.

Description: dark colored, twin woven hair-like fossil trichome.

Dimensions: holotype - total length $=164 \mu \mathrm{m}$; paratype - total length $=458 \mu \mathrm{m}$; range of total length $=164(253) 458 \mu \mathrm{m}(4$ specimens).

Derivation of name: latin duplihelicoidus - meaning double helicoid. Botanical affinity: unknown.

Trichomites simplex sp. nov.

Fig. $11 \mathrm{~A}-\mathrm{F}$

2010 'Tricoma simples delgado' in Arai \& Duarte, fig. 3A-3E (p. 181).

Holotype: Slide UNESP-4.1, D55/4 (Fig. 11C).

Type locality: Fazenda Nossa Senhora de Fátima (Ibaté municipality, São Paulo State, Brazil. UTM 23 7577,6 km, 197,75 km).

Type stratum: São Carlos Formation, FNSF Mbr, Ibaté Bed, upper Santonian.

Description: dark colored simple hair-like fossil trichome without spines or branches.

Dimensions (holotype): total length $=267 \mu \mathrm{m}$; diameter near base $=$ ca. $4.5 \mu \mathrm{m}$; range of total length $=219$ (350) $450 \mu \mathrm{m}$ (6 specimens).
Derivation of name: latin simplex - meaning simple.

Botanical affinity: several dicotyledon families possess simple hairlike trichomes - e.g., Brassicaceae, Fabaceae and Rutaceae (Metcalfe \& Chalk, 1988).

\section{Palynostratigraphy and age of the Ibaté Bed}

Several pollen taxa indicate that the studied samples are confined to the Coniacian - Santonian chronostratigraphic interval (89.8-83.6 Ma, ICS, 2017). Some of the pollen taxa belong exclusively to this interval such as Anacolosidites eosenonicus, Foveotricolpites gigantoreticulatus, F. tienabaensis and Victorisporis robertii (Fig. 15). Lima et al. (1986) already gave a similar Coniacian chronostratigraphic assignment for samples from an "equivalent section" (from another close-by stream in the same area). The material studied by those authors contains similar palynological assemblage to that found in the Ibate Bed. They mentioned the following common species: cf. Accuratispollis sp. (= Anacolosidites eosenonicus), Classopollis classoides, Confossia vulgaris, Constantinisporis jacquei, Cretacaeiporites polygonalis, Gabonisporis vigourouxii, Gnetaceaepollenites jansonii, Hexaporotricolpites emelianovi, Tricolpites tienabaensis (= Foveotricolpites tienabaensis), Victorisporis roberti and Zlivisporis blanensis. Nevertheless, their paper was limited to a short preliminary report, with no detailed qualitative and quantitative data, such as that given here.

Anacolosidites eosenonicus sp. nov. was firstly called "cf. Accuratipollis sp." by Ioannides and Colin (1977) who reported in the Coniacian-Santonian of the DSDP Site 356. Lima et al. (1986) and Azevedo et al., 1987 kept this provisional name, but considered this taxon as a guide-pollen for the Coniacian. It was renamed as Anacolosidites sp. in Viviers and Azevedo (1988), and frequently cited as "Anacolosidites sp. A" afterward. Its name was used to nominate the "Anacolosidites sp. interval zone" by the latter authors, and referred as Coniacian in age; its boundaries were defined based on the last occurrences (LOs) of Steevesipollenites nativensis (base) and Anacolosidites sp. (top).

Several marine sections drilled in offshore areas of the Campos and Santos basins, and calibrated with respect to foraminifera and nannofossil zones, indicate that the Anacolosidites sp. Zone, herein renamed as Anacolosidites eosenonicus Palynozone, is restricted to the upper Santonian (Arai et al., 2010). The top of the Anacolosidites eosenonicus Palynozone coincides with the first occurrence (FO) of Globotruncanita spp. (foraminifera) indicating the Santonian-Campanian boundary (Viviers et al., 2005) and occurs between the LOs of the nannofossil species Eprolithus moratus and Lithastrinus grilli that occur respectively in upper Santonian and in lower Campanian. Furthermore, the top of the Anacolosidites eosenonicus Palynozone is situated between the LOs of the dinoflagellate species Oligosphaeridium pulcherrimum and Nelsoniella aceras that occur respectively in the middle Santonian and in middle Campanian (Arai et al., 2010). The absence of Steevesipollenites nativensis in the Ibate Bed indicates that the interval in question is directly located within the Anacolosidites eosenonicus Palynozone. Therefore, we assign all studied samples to the upper Santonian (ca. 84.5-83.6 Ma). Considering data published by Dias-Brito et al. (2001), the Ibaté Bed is stratigraphically related to the uppermost part of the Adamantina and Uberaba formations (Bauru Group units). This Uberaba Formation was considered close to $83 \mathrm{Ma}$ and Santonian in age (Dias-Brito et al., 2001, p. 284, 288).

\section{Paleoenvironmental significance}

Among terrestrial palynomorphs, ephedroid pollen (Ephedratype pollen) such as Equisetosporites, Gnetaceaepollenites and Steevesipollenites have consistent abundance (Fig. 16; Supplementary 


\begin{tabular}{|c|c|c|c|c|c|c|c|}
\hline \multirow{2}{*}{ SPECIES } & \multirow{2}{*}{$\begin{array}{l}\text { EARLY } \\
\text { CRET. } \\
\text { ALBIAN } \\
\end{array}$} & \multicolumn{6}{|c|}{ LATE CRETACEOUS } \\
\hline & & CENOMAN. & TURONIAN & CONIACIAN & SANTONIAN & CAMPANIAN & MAASTR. \\
\hline \multicolumn{8}{|l|}{ Anacolosidites eosenonicus } \\
\hline \multicolumn{8}{|l|}{ Confossia vulgaris } \\
\hline \multicolumn{8}{|l|}{ Cretacaeiporites polygonalis } \\
\hline \multicolumn{8}{|l|}{ Cretacaeiporites scabratus } \\
\hline \multicolumn{8}{|l|}{ Foveotricolpites gigantoreticulatus } \\
\hline \multicolumn{8}{|l|}{ Foveotricolpites tienabaensis } \\
\hline \multicolumn{8}{|l|}{ Gabonisporis vigourouxii } \\
\hline \multicolumn{8}{|l|}{ Hexaporotricolpites emelianovi } \\
\hline \multicolumn{8}{|l|}{ Psilastephanoporites brasiliensis } \\
\hline \multicolumn{8}{|l|}{ Retitricolporites belmontensis } \\
\hline \multicolumn{8}{|l|}{ Victorisporis roberti } \\
\hline \multicolumn{8}{|l|}{ Zlivisporis blanensis } \\
\hline Steevesipollenites nativensis & & & & & & & \\
\hline
\end{tabular}

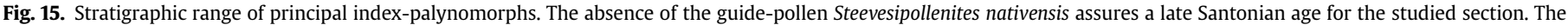
interval pointed out by hatched signature corresponds to the inferred age of the Ibate Bed.

Table A). Their occurrence associated with Classopollis grains suggest a warm climate tending towards dryness, as pointed out by the assumption of several authors (e.g., Srivastava, 1968; Arai and Coelho, 2001; Naváez et al., 2014). This must be the paleoclimatic condition that prevailed at the studied area during the late Santonian. The trilete spores, also frequently present throughout the studied section, indicate that some more humid phases occurred during this time. As the proportions of the palynomorph groups mentioned are inversely correlated, it is possible to follow the trends of the climatic fluctuations. The increase of pteridophyte spores may reflect a lacustrine expansion phase, associated with more humid conditions that favored the development of lakeside vegetation. Gabonisporis vigourouxii is related to the aquatic fern family Marsileaceae, as suggested by studies of fossil and extant Marsileaceae spores (e.g., Lupia et al., 2000; Takahashi et al., 2001; Schneider \& Pryer, 2002). Since Gabonisporis vigourouxii is one of most frequent spore species, this suggests a flourishing lakeside pteridophytic flora.

Some samples show abundant occurrence of algal elements. When only one type of microalgal remains prevails over other algal types, it is suggestive of phytoplankton blooms in the lake caused by eutrophication (Diersing, 2009). Botryococcus, Pediastrum, Staurastrum and prasinophyte remains were recognizable under fluorescence microscopy (Fig. 9I, K, M, O, Q S). The quantitative distribution of microalgal remains indicates that, when Staurastrum dominates, the frequencies of Botryococcus and Pediastrum are low (Fig. 16). There is often an inverse relationship between Botryococcus and Pediastrum (e.g., Van der Zwan \& Brugman, 1999; Shumilovskikh et al., 2014). According to Van der Zwan \& Brugman (1999), the dominance of Pediastrum may represent a true freshwater phase in the lake, whereas the dominance of Botryococcus can reflect brackish conditions. This shift in microalgal dominance is probably due to climatic variations. Indeed, the abundance of Pediastrum is only reported in samples with a relatively high proportion of pteridophyte spores (e.g., sample SC-14), which is interpreted to reflect episodic lacustrine expansions.
Prasinophytes (Leiosphaeridia spp.) seem also be incompatible with Botryococcus, but their presence is quite independent of Pediastrum, since we observe that prasinophyte peaks can coincide with those of Pediastrum (e.g., as in the sample SC-8) or not (e.g., as in the sample UN-5, where prasinophyte peak occurs in the absence of Pediastrum). The strata containing more Botryococcus than Pediastrum and Prasinophytes were likely deposited under low oxygen level in water body as suggested by Guy-Ohlson (1992) and Rodríguez-Amenábar \& Ottone (2003).

From the combination of sedimentological, geochemistry and palynological data, we interpret the Ibaté Bed as a deposit formed in a distal lake with episodic low-oxygen/anoxic conditions in bottom waters.

\section{Concurrence with the Great Santonian Wildfire}

The so-called "Great Santonian Wildfire" was first recorded from areas of the Santos and Campos offshore basins in the western South Atlantic by Arai et al. (2006) and has been interpreted to be a series of recurrent events associated with volcanic activity. Strata coeval to this event present abundant charred and/or burnt organic particles. The most curious aspect in these strata is the coexistence of well-preserved palynomorphs with carbonized organic matter that suggests vegetation recoveries in the intervals between fire events (Fig. 17). Furthermore we have observed conspicuous presence of paleowildfire indicators such as charcoals (Fig. 14E), astrosclereids (Stellatia spp.: Fig. 10) and trichomes (Trichomites spp.: Fig. 11) within the Ibaté Bed which was deposited during late Santonian, when the fire climax apparently took place. The Ibaté Bed section, which is located about $300 \mathrm{~km}$ from the Santos Basin littoral, contains sclereids/trichomes that are systematically associated with a rich palynological assemblage. This differs from the situation observed in the offshore basins, where the strata bearing abundant sclereids and/or trichomes are virtually barren in palynomorphs. Taking into account the distance separating São Carlos 


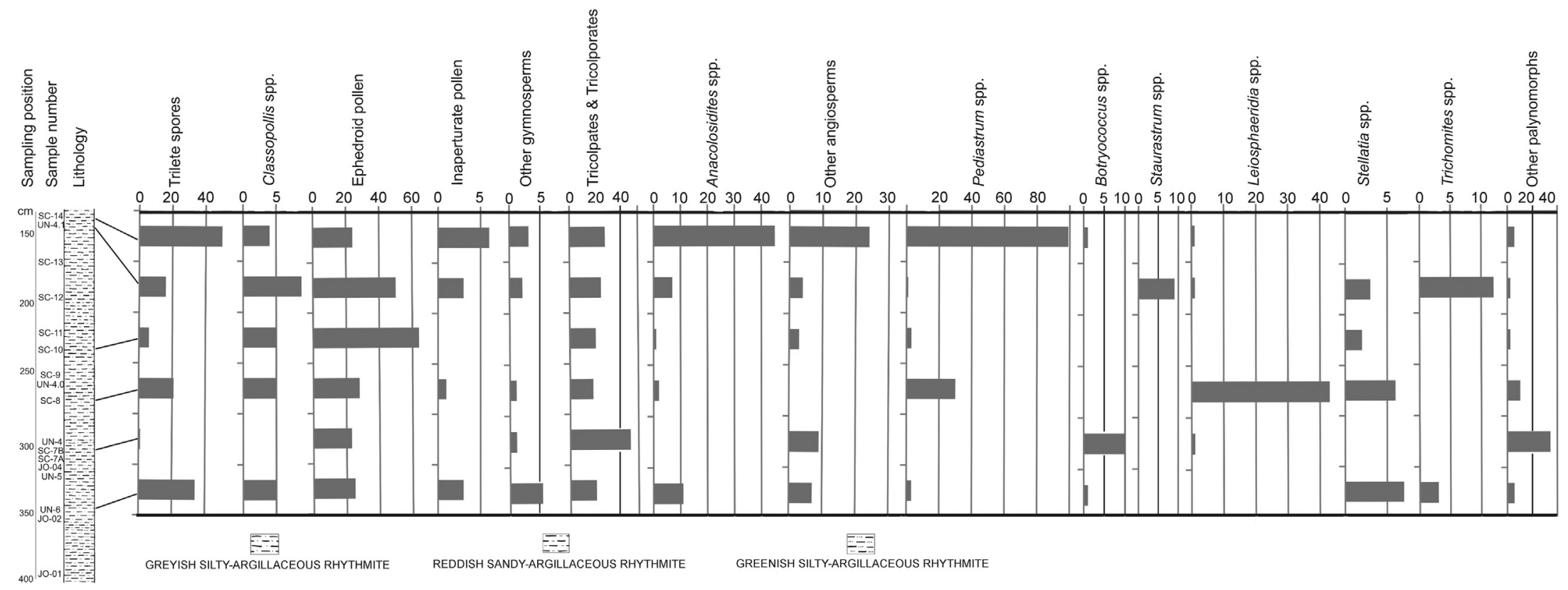

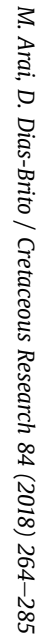

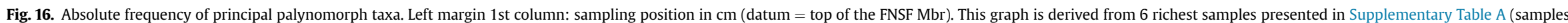
UNESP-6, SC-7B, SC-8, SC-10, UNESP-4.1 and SC-14). 

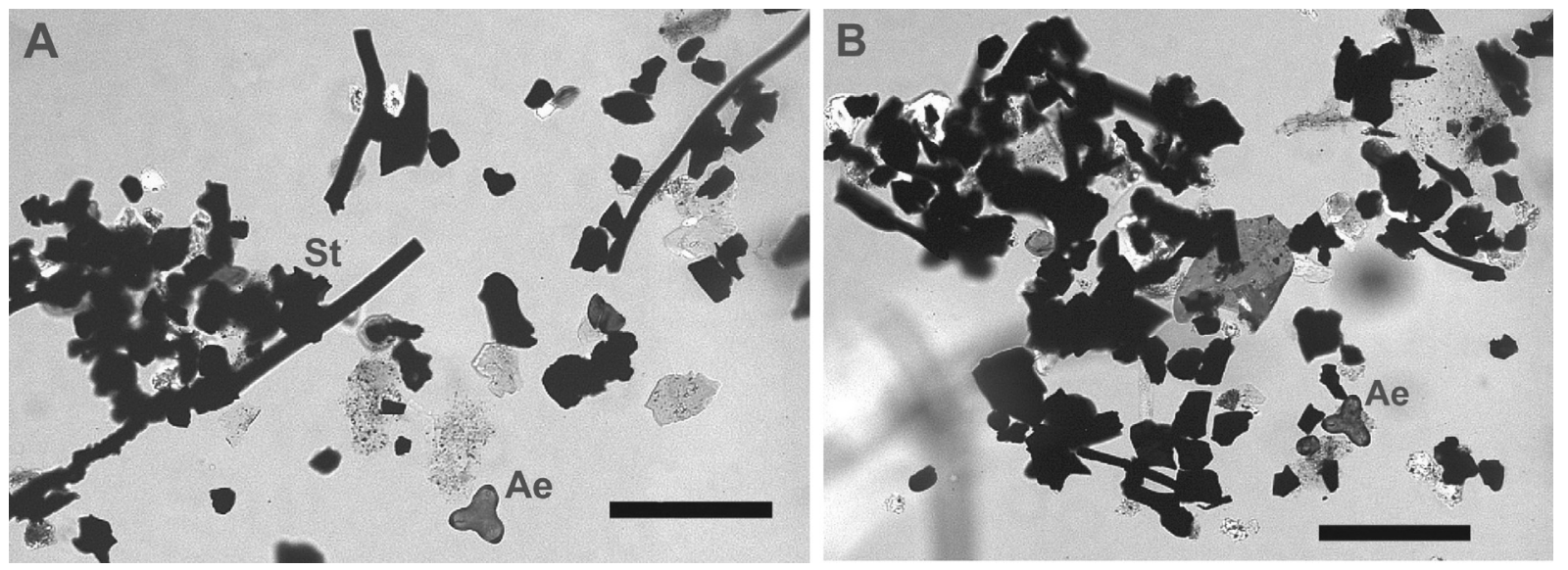

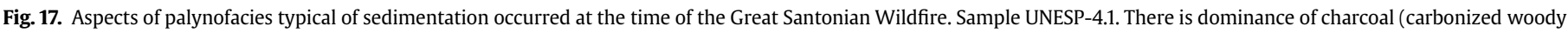

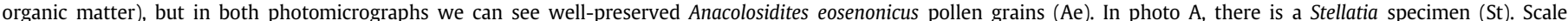
bars $=100 \mu \mathrm{m}$

and the coastal area, it is more plausible to consider that the wildfire signs recorded from the Ibate Bed were associated with volcanic activity that occurred in the interior of the country, an area located around $100 \mathrm{~km}$ away from the São Carlos Formation type section (Coutinho et al., 1982; Dias-Brito et al., 2001).

\section{Paleophytogeography}

In terms of Late Cretaceous paleophytogeographic frameworks, the palynoflora studied here stems from a tropical paleomicrofloristic region that would correspond more or less to the Senonian Palmae Province (cf. Herngreen \& Chlonova, 1981; Herngreen et al.,
1996). Nevertheless, pollen grains typically derived from Palmae e.g., Longapertites, Mauritiidites and Spinizonocolpites - are absent. On the other hand, the Ibaté Bed palynological content is closer to the pollen spectra from the middle Cretaceous ASA (African-South American) Province (Herngreen \& Chlonova, 1981). This is due to the fact that several palynomorph species recovered are typical of the ASA Province, such as Cretacaeiporites polygonalis, C. scabratus, Gnetaceaepollenites jansonii, Hexaporotricolpites emelianovi and Psilastephanoporites brasiliensis which survived since middle Cretaceous. Palynofloras from west and northeast Africa share many common species (Supplementary Table B) with the studied section. Despite the geographic proximity, time-equivalent

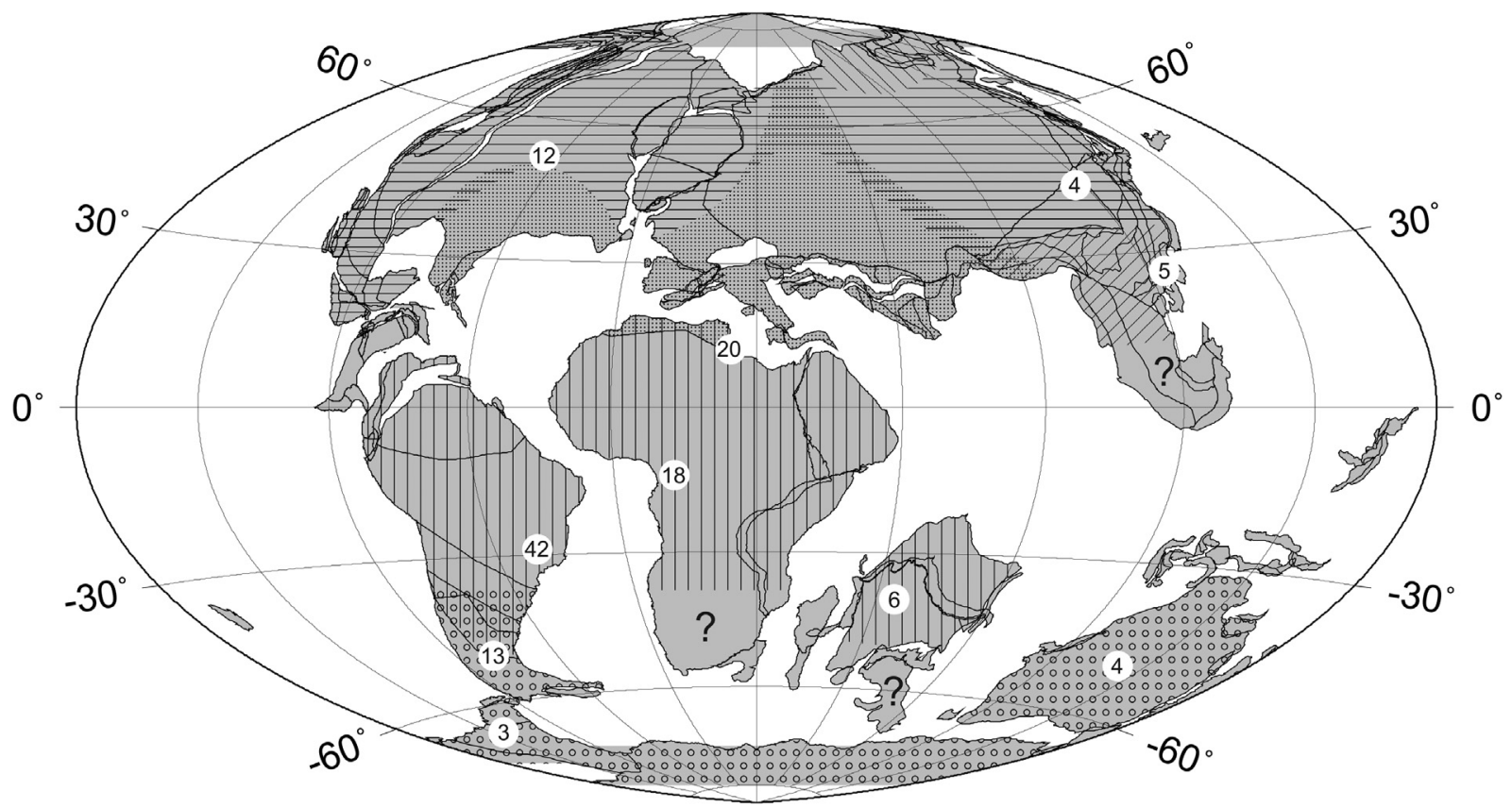

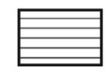

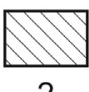

2

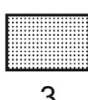

3
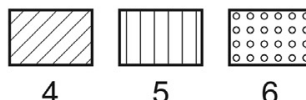

6

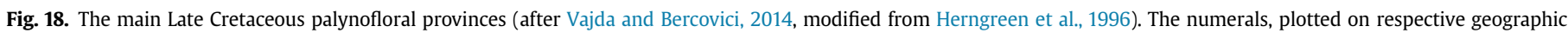

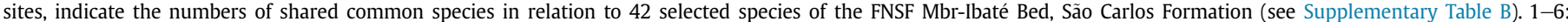

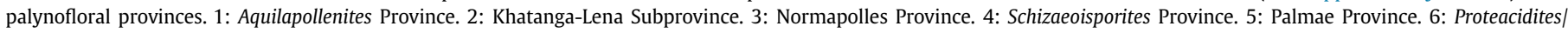
Nothofagidites Province. 
palynofloras from Argentina belong to another phytogeographic province and contain relatively few species in common with our material. This low number of common Cretaceous species between Argentina and São Carlos-Ibaté may be due to the incomplete nature of the upper Cenomanian-lower Campanian palynological record in Argentina, as indicated by Prámparo (2012). Herngreen et al. (1996) also assigned India to the Palmae Province, but in fact there are very few shared species when compared with the São Carlos-Ibaté record. The same is observed with respect to Antarctica, Asia, Australia and Europe. Besides the distance, these latter areas belong to other provinces, and were characterized by distinct paleoclimates. In order to show the similarity of the Ibaté Bed palynoflora with several Late Cretaceous palynofloras in the world, the Ibaté Bed assemblage was compared with those from 9 other palynofloristic provinces (Fig. 18).

\section{Conclusions}

The rich palynological content present in dark laminae of lacustrine silty-argillaceous rhythmites of the Ibaté Bed, FNSF Member, São Carlos Formation, previously dated as Coniacian-Santonian, is indicative of a late Santonian age. This new age assignment is based on palynostratigraphic relationships established from a reliable biostratigraphic framework based on integration of palynological, foraminiferal, calcareous nannofossil and dinocyst data.

Well-preserved palynomorphs are in the Ibaté Bed and represent the richest Upper Cretaceous palynological assemblage registered so far from a continental basin in Brazil. These deposits are stratigraphically related to the uppermost part of the Adamantina and Uberaba formations (stratigraphic units of the Bauru Group).

The predominance of ephedroid pollen associated with Classopollis grains is indicative of a warm climate tending towards dryness at the study area during the late Santonian. However, some more humid phases occurred during this time. The conspicuous occurrence of freshwater algal remains indicates that the Ibate Bed rhythmites accumulated in a permanent aquatic environment. From the combination of sedimentological, geochemical and palynological data, we propose that the deposition occurred in a distal lake, frequently with episodic low-oxygen/anoxic conditions in lake bottom water. Therefore, at this time, the area would have been a very particular environment within the vast continental territory represented by the "Bauru Basin".

The presence of charcoals, astrosclereids (Stellatia spp.) and trichomes (Trichomites spp.) in the Ibaté Bed indicates that the late Santonian vegetation of this region was affected by wildfires. Some of the wildfire activity may have been associated with important volcanic activity occurring not far from there (around $100 \mathrm{~km}$ ), which would have been coeval to the Great Santonian Wildfire recorded in coastal areas of some offshore basins in southeastern Brazil.

In general, among terrestrial palynomorphs, we observed a greater abundance of miospores derived from pteridophytes and gymnosperms, but, in terms of taxonomic diversity, angiosperm pollen represents the richest group. This could mean that the Santonian represented the beginning of the great expansion of the angiosperm group that occurred in the Late Cretaceous.

The exceptional late Santonian Ibaté Bed palynoflora is located at a transitional position between the middle Cretaceous ASA Province and the Senonian Palmae Province.

\section{Acknowledgements}

We are grateful to Rodrigo H. Goya and Deryk Willyan Biotto (UNESPetro) for support in making drawings for some of the figures; to Hermes Dias Brito (UNESPetro) for drawings and SEM images; to Mercedes B. Prámparo (IANIGLA/CONICET) for providing important publications about Argentinian palynology; to Valeria Perez Loinaze (Museo Argentino de Ciencias Naturales "Bernardino Rivadavia") for the revision and suggestions; to three anonymous referees for the revision of the manuscript; and to Peter Homewood, for checking the written English.

\section{References}

Agasie, J.M., 1969. Late Cretaceous palynomorphs from northeastern Arizona. Micropaleontology 15 (1), 13-30.

Akyuz, I., Warny, S., Famubode, O., Bhattacharya, J.P., 2015. Palynology of the Upper Cretaceous (Turonian) Ferron Sandstone Member, Utah, USA: identification of marine flooding surfaces and Milankovitch cycles in subtropical, ever-wet, paralic to non-marine palaeoenvironments. Palynology 40 (1), 122-136. Online version published on 29 jul 2015 at: http://sites01.lsu.edu/faculty/swarny/wpcontent/uploads/sites/30/2015/05/Akyuz-et-al.-2015.pdf.

Alley, N.F., Clarke, J.D.A., 1992. Stratigraphy and palynology of Mesozoic sediments from the Great Australian Bight area, southern Australia. BMR Journal of Australian Geology \& Geophysics 13, 113-129.

Arai, M., Coelho, P.S.M., 2001. Statistical analysis of palynological assemblages from the Aptian-Albian of the Araripe Basin, northeast Brazil: a case study of paleoenvironmental significance of Early Cretaceous terrestrial palynomorphs. In: Proceedings of the $7^{\text {th }}$ International Symposium on Mesozoic Terrestrial Ecosystems (Buenos Aires, September 26 - October 1, 1999), pp. 25-28 (Asociación Paleontológica Argentina, Buenos Aires, Publicación Especial 7).

Arai, M., Duarte, S.G., 2010. Tricomas fósseis como nova categoria de palinomorfos lato sensu: sua classificação preliminar e aplicabilidade na Bioestratigrafia. Boletim Museu Paraense Emílio Goeldi, Ciências Naturais 5 (2), 175-188.

Arai, M., Lana, C.C., Araújo, C.V., Menezes, T.R., 2006. O grande incêndio do Santoniano: considerações geológicas e exploratórias. Boletim de Geociências da Petrobras 14 (1), 125-131.

Arai, M., Viviers, M.C., Shimabukuro, S., 2010. Caracterização bioestratigráfica dos reservatórios cretáceos da Bacia de Santos. Petrobras/CENPES/PDGEO/BPA, Rio de Janeiro, 81 pp. (Internal Report RT BPA 007/10, Unpublished results).

Archangelsky, S., Bellosi, E.S., Jalfin, G.A., Perrot, C., 1994. Palynology and alluvial facies from the mid-Cretaceous of Patagonia, subsurface of San Jorge Basin, Argentina. Cretaceous Research 15, 127-142.

Archangelsky, A., Archangelsky, S., Poiré, D.G., Canessa, N.D., 2008. Registros palinológicos en la Formación Piedra Clavada (Albiano) en su area tipo, provincia de Santa Cruz, Argentina. Revista del Museo Argentino de Ciencias Naturales 10 (2), 185-198.

Askin, R.A., 1990. Cryptogam spores from the upper Campanian and Maastrichtian of Seymour Island, Antarctica. Micropaleontology 36 (2), 141-156.

Azevedo, R.L.M., Gomide, J., Viviers, M.C., Hashimoto, A.T., 1987. Bioestratigrafia do Cretáceo marinho da Bacia de Campos, Brasil. Revista Brasileira de Geociências 17 (2), 147-153.

Barreda, V., Palamarczuk, S., Medina, F., 1999. Palinología de la Formación Hidden Lake (Coniaciano-Santoniano), Isla James Ross, Antártida. Revista Española de Micropaleontología 31 (1), 53-72.

Batterman, M.R.W., Lammers, T.G., 2004. Branched foliar trichomes of Lobelioideae (Campanulaceae) and the infrageneric classification of Centropogon. Systematic Botany 29 (2), 448-458.

Belsky, C.Y., Boltenhagen, E., Potonié, R., 1965. Sporae dispersae der Oberen Kreide von Gabun, Äquatoriales Afrika. Paläontologische Zeitschrift 39 (1/2), 72-83, 2 plates.

Bettar, I., Meon, H., 2006. La palynoflore continentale de l'Albien du basin d'AgadirEssaouira (Maroc). Revue de Paléobiologie, Genève 25 (2), 593-631.

Braman, D.R., 2001. Terrestrial palynomorphs of the upper Santonian-?lower Campanian Milk River Formation, southern Alberta, Canada. Palynology 25, 57-107.

Castro, J.C., Dias-Brito, D., Arai, M., Rodrigues, R., Musacchio, E.A., 2002. Formação são Carlos: uma nova unidade para o Grupo Bauru (Cretáceo Continental do Brasil). In: Castro, J.C., Dias-Brito, D.R., Musacchio, E.A., Rohn, R. (Eds.), Boletim do VI Simpósio sobre o Cretáceo do Brasil. UNESP, São Pedro, pp. 351-357.

Cookson, I.C., Pike, K., 1954. Some dicotyledonous pollen types from Cainozoic deposits in the Australian region. Australian Journal of Botany 2, 197-219.

Coutinho, J.M.V., Coimbra, A.M., Brandt Neto, M., Rocha, G.A., 1982. Actas del $5^{\circ}$ Congresso Latino-Americano de Geologia, Argentina. Lavas alcalinas analcimíticas associadas ao Grupo Bauru (Kb) no Estado de São Paulo, Brasil, vol. II, pp. 185-195.

Deaf, A.S., Harding, I., Marshall, E.A., 2014. Cretaceous (Albian-?early Santonian) palynology and stratigraphy of the Abu Tunis $1 \mathrm{x}$ borehole, northern Western Desert, Egypt. Palynology 38 (1), 51-77.

Dettmann, M.E., 1963. Upper Mesozoic microfloras from south-eastern Australia Proceedings of the Royal Society of Victoria 77 (1), 1-148, plates 1-27.

Dettmann, M.E., Thomson, M.R.A., 1987. Cretaceous palynomorphs from the James Ross Island area, Antarctica - a pilot study. British Antarctic Survey Bulletin 77, 13-59.

Dias-Brito, D., Musacchio, E.A., Castro, J.C., Maranhão, M.S.A.S., Suárez, J.M., Rodrigues, R., 2001. Grupo Bauru: uma unidade continental do Cretáceo do 
Brasil - concepções baseadas em dados micropaleontológicos, isotópicos e estratigráficos. Revue de Paléobiologie 20 (1), 245-304.

Dias-Brito, D., Musacchio, E.A., Maranhão, M.S.A.S., Suárez, J.M., Castro, J.C., 2002 Micropaleontologia do Grupo Bauru em Neves Paulista-SP (Brasil) e uma pri meira proposta de partição estratigráfica da Formação Adamantina em dois intervalos microbióticos, In: Castro, J.C., Dias-Brito, D.R., Musacchio, E.A. Rohn, R. (Eds.), Boletim do VI Simpósio sobre o Cretáceo do Brasil. UNESP, São Pedro, pp. 335-343.

Diersing, N., 2009. Phytoplankton blooms: the basics. Florida Keys National Marine Sanctuary. Online version at: https://nmsfloridakeys.blob.core.windows.net floridakeys-prod/media/archive/scisummaries/wqpb.pdf.

Duarte, S.G., Arai, M., 2010. Esclerócitos: proposta de uma nova categoria de palinomorfos lato sensu. Revista Brasileira de Paleontologia 13 (1),67-76.

El-Beialy, S.Y., 1994. Palynostratigraphy and palynofacies analysis of some subsurface Cretaceous formations in the Badr El Dein (Bed 1-1) borehole, North Western Desert, Egypt. Neues Jahrbuch für Geologie und Paläontologie Abhandlungen 192 (2), 133-149.

Guy-Ohlson, D., 1992. Botryococcus as an aid in the interpretation of palaeoenvironment and depositional processes. Review of Palaeobotany and Palynology 71, 1-15.

Guy-Ohlson, D., 1996. Chapter 7B. Prasinophycean algae. In: Jansonius, J., McGregor, D.C. (Eds.), Palynology: Principles and Applications, vol. 1. American Association of Stratigraphic Palynologists, Dallas, pp. 181-189.

Herngreen, G.F.W., Chlonova, A.F., 1981. Cretaceous microfloral provinces. Pollen et Spores 23 (3-4), 441-555.

Herngreen, G.F.W., Kedves, M., Rovnina, L.V., Smirnova, S.B., 1996. Cretaceous palynofloral provinces: a review (chapter 29C). In: Jansonius, J., MacGregor, D.C. (Eds.), Palynology: principles and applications, vol. 3. American Association of Stratigraphic Palynologists Foundation, Dallas, pp. 1157-1188.

Ibrahim, M.I.A., Abdel-Kireem, M.R., 1997. Late Cretaceous palynofloras and foraminífera from Ain El-Wadi area, Farafra Oasis, Egypt. Cretaceous Research 18 633-660.

ICS-International Commission on Stratigraphy, 2017. International Chronostratigraphic Chart v. 2017/02. Online version at: http://www.stratigraphy.org/ ICSchart/ChronostratChart2017-02.pdf.

Ioannides, N.S., Colin, J.P., 1977. Palynology of sites 358, 356, 355, DSDP Leg 39, southwestern Atlantic Ocean. DSDP Initial Reports 39, 885-897. Online version at: http://deepseadrilling.org/39/volume/dsdp39_37.pdf.

Jardiné, S., Magloire, L., 1965. Palynologie et stratigraphie du Crétacé des bassins du Sénégal et de Côte d'Ivoire. Mémoire Bureau Recherche Géologie Mineralogie $32,187-245$.

Kumaran, K.P.N., Edet, J.J., 1995. Palynostratigraphy of the Late Cretaceous Nkporo Shale outcrop in the Anambra Basin, Nigeria. Palaeobotanist 42 (3), 372-379.

Li, W., Liu, Z., 1994. The Cretaceous palynofloras and their bearing on stratigraphic correlation in China. Cretaceous Research 15, 333-365.

Lima, M.R., Mezzalira, S., Dino, R., Saad, A.R., 1986. Descoberta de microflora em sedimentos do Grupo Bauru, Cretáceo do Estado de São Paulo. Revista do Instituto Geológico 7 (1/2), 5-9.

Lupia, R., Schneider, H., Moeser, G.M., Pryer, K.M., Cranes, P.R., 2000. Marsileacea sporocarps and spores from the Late Cretaceous of Georgia U.S.A. International Journal of Plant Sciences 161 (6), 975-988.

Mahmoud, M.S., 2003. Palynology and palaeoenvironment of the Quseir Formation (Campanian) from central Egypt. Journal of African Earth Sciences 36, 135-148.

Malécot, V., Lobreau-Callen, D., 2005. A survey of species assigned to the fossil pollen genus Anacolosidites. Grana 44, 314-336.

Metcalfe, C.R., Chalk, L., 1988. Anatomy of the Dicotyledons, v.1 - Systematic anatomy of leaf and stem, with a brief history of the subject, 276 pp., 18 plates, second ed. Clarendon Press, Oxford.

Mezzalira, S., 1974. Contribuição ao conhecimento da estratigrafia e paleontologia do Arenito Bauru. Instituto Geográfico e Geológico, São Paulo, 163 pp., 2 pls. map (Boletim IGG 51).

Mezzalira, S., 1989. Os fósseis do Estado de São Paulo. Instituto Geológico, Secretaria do Meio Ambiente, São Paulo, 136 p., 13 pls. (Série Pesquisa IG-SMA, second ed).

Miki, A., 1972. Palynological study of the Kuji Group in northern Honshu, Japan. Journal of the Faculty of Science, Series 4. Geology and Mineralogy 15 (3-4), $513-604$

Miki, A., 1973. Spores and pollen flora from the Middle Yeso Group in northern Hokkaido, Japan. Chishitsugaku Zasshi 79 (3), 205-218 (in Japanese).

Morgan, R., 1978. Albian to Senonian palynology of Sites 364, Angola Basin. In: Bolli, H.M., et al. (Eds.), Initial Reports of the Deep Sea Drilling Project, vol. 40. U.S. Government Printing Office, Washington, pp. 915-951.

Nandi, B., 1990. Palynostratigraphy of Upper Cretaceous sediments, Meghalaya, northern India. Review of Palaeobotany and Palynology 65, 119-129.

Narváez, P.L., Sabino, I.F., 2008. Palynology of the Las Curtiembres Formation (Late Cretaceous, Salta Group Basin), Las Conchas Creek area, northwestern Argentina. Ameghiniana 45 (2), 473-482.

Narváez, P.L., Prámparo, M.B., Sabino, I.F., 2014. First palynologic record of the Cretaceous La Yesera Formation (Salta Group), northwestern Argentina, Revista Brasileira de Paleontologia 17 (2), 141-156.

Potonié, R., 1960. Synopsis der Gattungen der Sporae dispersae. III. Teil: Nachträge Sporites, Fortsetzung Pollenites mit Generalregister zu Teil I - III. Beihefte zum Geologischen Jahrbuch 39, 1-189.

Prámparo, M.B., 2012. Non-marine Cretaceous palynomorph biostratigraphy of Argentina: a brief summary. Journal of Stratigraphy 36 (2), 213-228.
Prámparo, M.B., Volkheimer, W., 2002. Nuevos hallazgos de palinomorfos em la Formación La Amarga, Miembro Bañados de Caichigüe, cuenca Neuquina sudoriental, Argentina. Ameghiniana 39 (4), 395-404.

Premaor, E., 2016. Bioestratigrafia das associações de cistos de dinoflagelados do Cretáceo Inferior (Albiano) ao Neógeno (Plioceno) da Bacia de Pelotas, Brasil. Ph.D. Thesis, vol. 2. Universidade Federal do Rio Grande do Sul, Porto Alegre (Documentação Palinológica), 128 pp.

Quattrocchio, M.E., Martínez, M.A., Carpinelli Pavisich, A., Volkheimer, W., 2006. Early Cretaceous palynostratigraphy, palynofacies and palaeoenvironments of well sections in northeastern Tierra del Fuego, Argentina. Cretaceous Research 27, 584-602.

Regali, M.S.P., Uesugui, N., Santos, A.S., 1974a. Palinologia dos sedimentos mesocenozóicos do Brasil. I. Boletim Técnico da Petrobras 17 (3), 177-191.

Regali, M.S.P., Uesugui, N., Santos, A.S., 1974b. Palinologia dos sedimentos mesocenozóicos do Brasil. II. Boletim Técnico da Petrobras 17 (4), 263-301.

Rodríguez-Amenábar, C. Ottone, E.G., 2003. La aplicación de Botryococcus (Chlorococcales) como indicador paleoambiental em el Triásico de Argentina. Revista Española de Micropaleontología 35 (2), 161-169.

Rohn, R., Yan-bin, S., Dias-Brito, D., 2005. A new Coniacian-Santonian conchostracan genus from the Bauru Group, south-east Brazil: taxonomy, palaeobiogeography and palaeoecology. Cretaceous Research 26, 581-592.

Samant, B., Bajpai, S., 2005. Palynoflora from the Lakshmipur intertrappean deposits of Kutch, Gujarat: age implications. Journal of the Palaeontological Society of India, Golden Jubilee Volume 50 (2), 169-176.

Schneider, H., Pryer, K.M., 2002. Structure and function of spores in the aquatic heterosporous fern family Marsileaceae. International Journal of Plant Sciences 163 (4), 485-505.

Schrank, E., 1987. Biostratigraphic importance of microfloras from the Late Cretaceous Clastic Series of northwestern Sudan. Cretaceous Research 8, 29-42.

Shumilovskikh, L.S., Fleitmann, D., Nowaczyk, N.R., Behling, H., Marret, F., Wegwerth, A., Arz, H.W., 2014. Orbital- and millennial-scale environmental changes between 64 and 20 ka BP recorded in Black Sea sediments. Climate of the Past 10, 939-954.

Song, Z., Li, M., Wang, W., Zhao, C., Zhu, Z., Zheng, Y., Zhang, Y., Wang, D., Zhou, S. Zhao, Y., 1999. Fossil spores and pollen of China, v.1 - The Late Cretaceous and Tertiary Spores and Pollen, 910 pp., 207 plates. Science Press, Beijing.

Srivastava, S.K., 1967. Palynology of Late Cretaceous mammal beds, Scollard, Alberta (Canada). Palaeogeography, Palaeoclimatology, Palaeoecology 3, 133-150.

Srivastava, S.K., 1968. Ephedralean pollen from the Upper Cretaceous Edmonton Formation of Alberta (Canada) and their paleoecological significance. Canadian Journal of Earth Sciences 5, 211-221.

Takahashi, K., Sugiyama, R., 1990. Palynomorphs from the Santonian Uge Member of the Taneichi Formation, Northeast Japan. Bulletin Faculty of Liberal Arts, Nagasaki University (Natural Science) 30 (2), 133-573.

Takahashi, M., Crane, P.R., Ando, H., 2001. Fossil megaspores of Marsileales and Selaginellales from the upper Coniacian to lower Santonian (Upper Cretaceous) of the Tamagawa Formation (Kuji Group) in Northeastern Japan. International Journal of Plant Sciences 162 (2), 431-439.

Uesugui, N., 1979. Palinologia: técnicas de tratamento de amostras. Boletim Técnico da Petrobras 22 (4), 229-240.

Vajda, V., Bercovici, A., 2014. The global vegetation pattern across the CretaceousPaleogene mass extinction interval: a template for other extinction events. Global and Planetary Change 122, 29-49.

Vallati, P., 2006. Las primeras angiospermas en el Cretácico de la Cuenca Neuquina (centro oeste de Argentina): aspectos geológicos relacionados. Revista Brasileira de Paleontologia 9 (1), 83-92.

Vallati, P., 2010. Asociaciones palinológicas con angiospermas en el Cretácico Superior de la Cuenca Neuquina, Argentina. Revista Brasileira de Paleontologia 13 (2), 143-158.

Vallati, P., 2013. Paleotropical pollen grains from the Neuquén Group, Patagonia, Argentina. Carnets de Géologie, Letter 2013/05 (CG2013_L05) 273-279.

Van der Zwan, C.J., Brugman, W.A., 1999. Biosignals from the EA Field, Nigeria. In: Jones, R.W., Simmons, M.D. (Eds.), Biostratigraphy in Production and Development Geology. Geological Society, London. Special Publications 152, 291-301.

Viviers, M.C., Azevedo, R.L.M., 1988. The Southeastern área of the Brazilian Continental Margin: its evolution during the middle and Late Cretaceous as indicated by paleoecological data. Revista Brasileira de Geociências 18 (3), 291-298.

Viviers, M.C., Ferreira, E.P., Lana, C.C., Arai, M., Shimabukuro, S., Beurlen, G., 2005. Biocronoestratigrafia integrada e evolução paleoambiental da seção albiana-maastrichtiana da Bacia de Santos: mapas paleobatimétricos. Petrobras/ CENPES/PDEX/BPA, Rio de Janeiro, 111 pp. (Internal Report RT BPA 04/2005, Unpublished results).

Volkheimer, W., Caccavari de Filice, M.A., Sepulveda, E., 1977. Datos palinológicos de la Formación Ortiz (Grupo La Amarga), Cretácico Inferior de la Cuenca Neuquina (República Argentina). Ameghiniana 14 (1-4), 59-74.

Wall, D., 1965. Evidence from recent plankton regarding the biological affinities of Tasmanites Newton 1875 and Leiosphaeridia Eisenack 1958. Geological Magazine 99 (4), 353-363.

\section{Appendix A. Supplementary data}

Supplementary data related to this article can be found at https://doi.org/10. 1016/j.cretres.2017.11.014. 